\title{
Formulation pseudo-3D de la diffusion du champ magnétique dans un rotor ferromagnétique massif. Application à l'étude des effets d'extrémité
}

\author{
Jean-Marie Biedinger \\ Laboratoire d'électromécanique, Université de Compiègne, BP 649, 60206 Compiègne Cedex, France
}

(Reçu le 10 juillet 1989, révisé le 11 janvier 1990, accepté le 12 mars 1990)

\begin{abstract}
Résumé. - Dans cet article on développe un modèle pseudo-3D pour résoudre en termes de potentiels $(\mathbf{A} ; \psi)$ le problème de la diffusion du champ magnétique dans un rotor ferromagnétique massif en régime stationnaire harmonique : a) d'un point de vue physique, ce modèle est qualifié de 3D car il prend en compte la nature vectorielle des grandeurs du champ électromagnétique; b) d'un point de vue géométrique, il est qualifié de pseudo-3D car il s'appuie sur une modélisation géométrique $2 \mathrm{D}$, la troisième dimension étant intégrée explicitement après homogénéisation de la structure suivant la direction circonférentielle; c) la restriction au régime stationnaire harmonique permet d'utiliser la représentation complexe pour traiter implicitement la dimension temps; d) la définition d'une perméabilité magnétique équivalente permet de prendre en compte la saturation magnétique du rotor sans affecter l'isotropie circonférentielle. L'intérêt du modèle pseudo-3D est de fournir une approche complémentaire des modèles $2 \mathrm{D}$, spécialisée pour l'étude des phénomènes liés aux anisotropies axiales dans les actionneurs à rotor massif. Cela devrait permettre de réserver l'utilisation des logiciels $3 \mathrm{D}$ à des configurations particulières d'actionneurs, quand les anisotropies circonférentielles et axiales de structure sont trop fortement couplées pour pouvoir en séparer les effets. A titre d'exemple, nous utilisons ce modèle pour préciser les effets d'extrémité dans un moteur asynchrone à rotor ferromagnétique massif.
\end{abstract}

\begin{abstract}
In this paper we present a quasi-3D finite element model for solving the nonlinear steady-state diffusion problem in a solid steel rotor. It is $3 \mathrm{D}$ qualified because it takes into account the three-dimensional nature of the electromagnetic field. However with regard to the geometry, it is pseudo-3D qualified because it is based on a two-dimensional $r$ - $z$ finite element mesh, with the third dimension being explicitly integrated after homogenization of the structure in the peripheral direction. By restricting ourselves to the harmonic steady state, we can use the complex phasor representation for solving the field equations. The definition of an equivalent permeability allows us to take into account the magnetic saturation without affecting the circumferential isotropy of the structure. The objective of this model is to provide a complementary approach to 2-dimensional models, suitable for the study of the influence of axial anisotropies in solid steel rotor machines. This would reserve the use of full 3-dimensional codes to specific machines, for which we cannot decouple axial and circumferential anisotropies. We will apply this model to the analysis of the finite-length effects in a solid steel rotor.
\end{abstract}

\section{Introduction.}

La recherche d'actionneurs mieux intégrés dans les chaînes de transfert d'énergie conduit à développer des moteurs asynchrones à rotor ferromagnétique massif. Pour ne retenir que les plus récents d'entre eux, on peut citer :

- le moteur rapide de puissance devant se substituer aux turbines dans les entraînements directs de pompes, compresseurs, ventilateurs [1],

- le moteur à deux degrés de liberté pour assurer le positionnement en translation et en rotation d'un mobile dont le rotor ferromagnétique fait partie intégrante [5].

Le développement de ces actionneurs nécessite des moyens d'investigations numériques spécifiques, avec des hypothèses de travail moins restrictives que celles utilisées par les modèles bidimensionnels classiques. En effet leur fonctionnement repose sur l'interaction entre des courants induits qui s'établissent dans des structures à géométrie tridimension- 
nelle et à loi de comportement magnétique non linéaire, et un champ inducteur dont la distribution dépend du nombre de degrés de liberté mécaniques pilotés.

Tout d'abord il faut insister sur le fait que le processus de diffusion du champ magnétique dépend fortement de la nature tridimensionnelle de l'objet dans lequel il s'exerce : les lignes de courant induit se développent préférentiellement à la périphérie du milieu conducteur, suivant des trajets influencés par sa topologie externe. Or celle-ci est fortement tributaire de contraintes d'origines très diverses :

- pour les moteurs rapides, les contraintes mécaniques imposent de dimensionner le contour frontal du rotor sans variation brusque [1] ; ceci exclut par exemple l'emploi de plaques d'extrémité conductrices qui contribueraient à mieux canaliser les lignes de courant actives,

- les pertes de surface nécessitent un rainurage circonférentiel du rotor, dimensionné pour filtrer les harmoniques de denture sans perturber la création de couple par le champ fondamental [2],

- le relèvement du facteur de puissance passe par un rainurage axial [3],

- des considérations d'inertie peuvent conduire à évider le rotor [4].

Ensuite il faut souligner l'influence de la saturation du milieu ferromagnétique qui modifie la répartition des lignes de courant et surtout rend les équations non linéaires. Ce phénomène intervient toujours dans ce type de rotor, même à faible niveau de champ d'entrefer, car la tendance des courants induits à se concentrer à la périphérie entraîne la modification du trajet des lignes d'induction, en les redistribuant sur une faible épaisseur dans la direction tangentielle.

Enfin il faut mentionner le rôle des sources du champ, que l'on confine habituellement au niveau de l'entrefer, mais qui peut également s'exprimer en dehors de la partie active : on connaît l'importance $\mathrm{du}$ problème des pertes supplémentaires dans les zones frontales des grosses machines, mais on peut également s'interroger sur le couplage entre les têtes de bobines et les extrémités du rotor. De plus la saturation peut imposer des couplages supplémentaires entre les différentes composantes du champ d'excitation : dans un moteur tubulaire axial à deux degrés de liberté par exemple, les champs inducteurs glissant et tournant ne sont plus tout à fait indépendants.

Les méthodes classiques d'investigation n'apportent pas toujours des réponses satisfaisantes aux problèmes posés par ce type d'actionneur.

L'approche analytique est très limitée dans les hypothèses de géométrie et de linéarité inhérentes à son développement.
Les modèles numériques bidimensionnels, à la base de logiciels largement éprouvés, permettent d'inclure la saturation magnétique et les anisotropies circonférentielles de structure. Mais, par nature, ils font abstraction des anisotropies axiales. La démarche classique consiste à corriger les modèles $2 \mathrm{D}$ avec des facteurs adaptés pour prendre implicitement en compte les perturbations apportées par la troisième dimension. Cette correction porte généralement sur la conductivité du rotor pour rendre compte de l'allongement du trajet des lignes de courant se refermant par les zones frontales. Elle peut s'avérer suffisante pour le calcul du couple dans certaines plages de fonctionnement, mais la confrontation avec l'expérimentation montre ses limites quant au calcul des grandeurs réactives [7]. Et encore faut-il au préalable déterminer ces facteurs correctifs. De toute façon, ils deviennent insuffisants pour donner une image correcte des variations de la topologie axiale quand il s'agit de résoudre des problèmes comme le dimensionnement du rainurage circonférentiel, ou le couplage des têtes de bobines avec les zones frontales.

Le développement de modèles numériques 3D [6] devrait permettre de donner une réponse au problème posé par les actionneurs à rotor massif : résoudre l'équation de diffusion non linéaire dans un milieu ferromagnétique conducteur en tenant compte des anisotropies de structure et de la topologie des sources. Mais ces modèles exigent un environnement informatique trop lourd pour prétendre constituer actuellement un outil de développement robuste qui soit adapté à de telles études. De plus ils ne sont vraiment nécessaires que dans la mesure où les anisotropies de structure sont trop fortement couplées pour ne pouvoir en séparer les effets (en général le processus de conversion d'énergie privilégie une direction qui peut être exploitée pour découpler les phénomènes).

Il nous a donc paru souhaitable de compléter les modèles 2D par un modèle qui privilégie les anisotropies axiales de structure au détriment des anisotropies circonférentielles. Les grandeurs physiques y conservent leur nature tridimensionnelle, mais leurs variations suivant la direction circonférentielle sont supposées telles que la coordonnée géométrique associée puisse être explicitement intégrée, réduisant ainsi l'espace géométrique à deux dimensions. Ce sont les modèles qualifiés de pseudo-3D.

Dans cet article on développe un modèle pseudo$3 \mathrm{D}$ de l'équation de diffusion non linéaire en régime stationnaire harmonique, restreint au cas d'un milieu conducteur électriquement homogène et simplement connexe. Ceci permet de baser le modèle sur une formulation mixte en potentiel vecteur magnétique dans la région conductrice, et en potentiel scalaire magnétique dans le reste du domaine d'étude. Les sources du champ sont introduites sous forme d'une 
nappe de courant équivalente, laquelle se traduit par une coupure sur le potentiel scalaire. La restriction au régime stationnaire harmonique permet d'utiliser la représentation complexe pour traiter implicitement la dimension temps. La définition d'une perméabilité magnétique équivalente permet d'introduire la saturation magnétique du rotor ferromagnétique sans détruire l'hypothèse temporelle du modèle sinusoïdal, tout en préservant l'isotropie circonférentielle de structure. Après avoir discuté de sa validité, nous utilisons ce modèle pour traiter des effets d'extrémité dans un moteur asynchrone à rotor massif.

\section{Formulation $3 D$ du problème de la diffusion non linéaire}

Les problèmes de diffusion en milieu conducteur sont régis par les équations de Maxwell dans l'approximation des états quasi stationnaires. Leur description en termes de potentiel vecteur magnétique $\mathbf{A}$ (induction magnétique $\mathbf{B}=\operatorname{rot} \mathbf{A}$ ) et de potentiel scalaire électrique $\Phi$ (champ électrique $\mathbf{E}=-\partial \mathbf{A} / \partial t-\operatorname{grad} \Phi)$ constitue une formulation mathématique intéressante pour la classe des problèmes concernés. Notons à cet effet que la formulation duale en $\mathbf{T}-\boldsymbol{\Omega}$ (densité de courant $\mathbf{J}=\operatorname{rot} \mathbf{T}$; excitation magnétique $\mathbf{H}=\mathbf{T}-\operatorname{grad} \Omega$ ) semble moins bien adaptée, le champ $\mathbf{H}$ se calculant comme la différence de deux grandeurs équivalentes, qui deviennent très grandes dans le cas d'un processus de diffusion caractérisé par une profondeur de pénétration petite devant la longueur d'onde des sources [8].

Dans le cas de grandeurs à variations temporelles sinusoïdales, synchrones d'une seule pulsation $\omega$, les équations gouvernant la répartition du champ s'écrivent :

$$
\begin{gathered}
\operatorname{rot}\left(\frac{1}{\mu_{\mathrm{e}}} \operatorname{rot} \underline{\mathbf{A}}\right)=-\gamma(j \omega \underline{\mathbf{A}}+\operatorname{grad} \underline{\Phi})+\underline{\mathbf{J}}_{s} \\
\operatorname{div} \gamma(j \omega \underline{\mathbf{A}}+\operatorname{grad} \underline{\Phi})=0
\end{gathered}
$$

où $\underline{\mathbf{A}}$ est $\underline{\Phi}$ sont les représentations complexes de $\mathbf{A}$ et $\Phi$ :

$$
\begin{gathered}
=\quad \mathbf{A} \times \mathrm{e}^{j \omega t} \\
\Phi(\mathbf{x}, t)=\text { Réel }\left\{\underline{\Phi}(\mathbf{x}) \mathrm{e}^{j \omega t}\right\}
\end{gathered}
$$

$\gamma$ la conductivité électrique, $\mathbf{J}_{\mathbf{s}}$ la densité de courant des sources, et $\mu_{\mathrm{e}}$ la perméabilité magnétique qu'il nous reste à définir dans le cas de matériaux magnétiques saturables en régime harmonique.

Mais ces équations ne sont pas suffisantes pour assurer l'unicité de $\underline{\mathbf{A}}$ et $\Phi$, car tout couple de fonctions $(\underline{\mathbf{A}}+\operatorname{grad} \underline{f})$ et $(\underline{\Phi}-j \omega \underline{f})$ vérifie encore (1) et (2). D'après le théorème d'Helmholtz [9], il faut également définir la divergence de $\underline{\mathbf{A}}$. Il est habituel d'utiliser la jauge de Coulomb :

$$
\operatorname{div} \underline{\mathbf{A}}=\mathbf{0}
$$

que l'on peut implémenter numériquement à l'aide des multiplicateurs de Lagrange [10], ou par modification de la formulation variationnelle [11]. Mais comme le potentiel n'a pas de signification physique en soi, on peut aussi utiliser un algorithme permettant la résolution de systèmes avec matrice singulière [12].

Or à l'intérieur d'un milieu conducteur homogène simplement connexe, le potentiel $\underline{\mathbf{A}}$ est suffisant pour décrire le champ électromagnétique ( $\underline{\mathbf{B}} ; \underline{\mathbf{E}})$. On peut alors faire abstraction du potentiel scalaire électrique $\Phi$, et montrer que dans ces conditions l'équation (1) détermine de façon univoque le potentiel $\underline{\mathbf{A}}$, sous réserve toutefois de spécifier sa valeur, ou son rotationnel, sur la surface du conducteur [13]. Le champ magnétique à l'extérieur du conducteur doit alors être décrit en termes de potentiel scalaire magnétique $\psi$, les conditions de passage du champ à la frontière du conducteur servant à spécifier $\underline{\mathbf{A}}$ ou rot $\underline{\mathbf{A}}$ en fonction de $\boldsymbol{\psi}$. Cette formulation mixte assure de plus un nombre minimal de degrés de liberté.

Ces considérations conduisent à la partition suivante du domaine d'étude (Fig. 1):

- La zone 1 ne contient aucun courant. Le champ d'excitation magnétique est déterminé en termes de potentiel scalaire $\psi: \underline{H}=-\operatorname{grad} \psi$.

- La zone 2 contient les courants sources que l'on modélisera comme une nappe de courant $\underline{\mathbf{J}}_{\mathrm{e}}$ d'épaisseur nulle, définie à partir du fondamental de la f.m.m. du bobinage réel. Elle se traduit par une coupure $\Delta \underline{\psi}$ sur le potentiel $\underline{\psi}$ le long de sa surface $S_{12}$, assurant ainsi son unicité.

- La zone 3 est limitée au milieu conducteur décrit par le potentiel $\underline{\mathbf{A}}$.

- Le couplage des zones 1 et 3 est réalisé en exprimant la continuité des composantes normale de $\underline{B}$ et tangentielle de $\underline{\mathbf{H}}$ sur leur surface commune $S_{13}$, en termes de potentiels $\underline{\mathbf{A}}$ et $\underline{\psi}$.

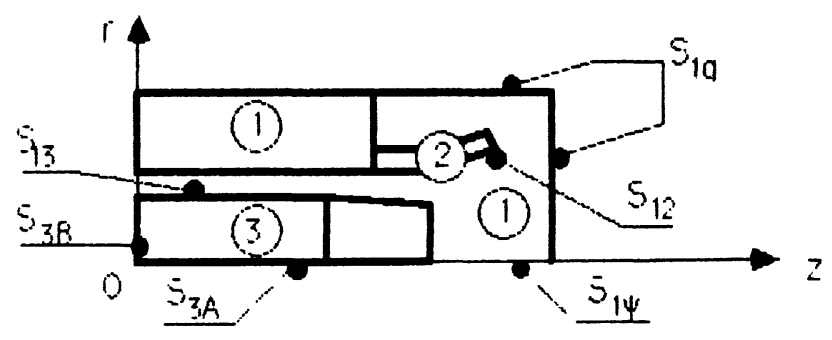

Fig. 1. - Partition du domaine: $1=\underline{\psi} ; 2=\Delta \underline{\psi}$; $3=\underline{\mathbf{A}}$

[Partitioning of the domain : $1=\underline{\psi} ; 2=\Delta \underline{\psi} ; 3=\underline{\mathbf{A}}$.] 
A cette partition correspond le système suivant d'équations :

FORMULATION DANS LA RÉGION NON CONDUCTRICE 1. - (Volume $V_{1}$; surface $S_{1}$; potentiel $\psi$ )

$$
\operatorname{div}\left(\mu_{1} \operatorname{grad} \underline{\psi}\right) \equiv 0
$$

$\operatorname{sur} S_{1 \psi}$ :

$$
\underline{\psi}=\underline{\psi}_{0} \quad \text { (axe de symétrie) }
$$

$\operatorname{sur} S_{1 \mathrm{q}}$ :

$$
\mu_{1} \partial \underline{\psi} / \partial n=0 \quad \text { (confinement de } \underline{\mathbf{B}} \text { dans } V_{1} \text { ) }
$$

$\operatorname{sur} S_{13}$;

$$
\underline{\mathbf{n}}_{13} \cdot\left(-\mu_{1} \operatorname{grad} \underline{\psi}\right)=\mathbf{n}_{13} \cdot \operatorname{rot} \underline{\mathbf{A}} .
$$

\section{FORMULATION SUR LA NAPPE DE COURANT.}

Sur $S_{12}$ :

$$
\begin{aligned}
& \Delta \underline{\psi}(b)=\underline{\psi}\left(b_{2}\right)-\underline{\psi}\left(b_{1}\right)=-\int_{a}^{b} \underline{\mathbf{J}}_{\mathrm{e}} \cdot \mathrm{d} \mathbf{l} \\
& \Delta \underline{\psi}(a)=0
\end{aligned}
$$

$a$ et $b \in S_{12} ; b_{1}$ et $b_{2}$ de part et d'autre de $b$ sur $S_{12}$.

FORMULATION DANS LA RÉGION CONDUCTRICE. (Volume $V_{3}$; surface $S_{3}$; potentiel $\underline{\mathbf{A}}$ )

$$
\operatorname{rot}\left(\frac{1}{\mu_{\mathrm{e}}} \operatorname{rot} \underline{\mathbf{A}}\right)+j \omega \underline{\mathbf{A}}=0
$$

$\operatorname{sur} S_{3 \mathrm{~A}}$ :

$$
\underline{\mathbf{A}}=0 \quad \text { (axe de symétrie) }
$$

$\operatorname{sur} S_{3 \text { в }}$ :

$$
\underline{\mathbf{A}} \wedge \mathbf{n}=0 \quad(\text { plan de symétrie contenant } \underline{\mathbf{B}})
$$

$\operatorname{sur} S_{13}$ :

$$
\mathbf{n}_{13} \wedge\left(\frac{1}{\mu_{3}} \operatorname{rot} \underline{\mathbf{A}}\right)=-\mathbf{n}_{13} \wedge \operatorname{grad} \underline{\psi}
$$

$\mu_{\mathrm{e}}=\mu_{\mathrm{e}}\left(\mu_{3}, \underline{\mathbf{B}}\right)=$ perméabilité magnétique équivalente.

\section{Remarque.}

La relation (2) n'étant pas prise explicitement en compte dans le système d'équations (5), (6), (7), il faut vérifier que leur résolution conduit bien à une répartition de densité de courants induits $\mathbf{J}$ conservative :

$$
\begin{aligned}
\operatorname{div} \underline{\mathbf{J}} & \equiv 0 \text { dans } V_{3} \\
\underline{\mathbf{J}} \cdot \mathbf{n} & \equiv 0 \text { sur } S_{3} .
\end{aligned}
$$

Alors que la première condition est contenue dans l'équation (7) :

$$
\begin{aligned}
\operatorname{div}\left[\operatorname{rot}\left(\frac{1}{\mu_{\mathrm{e}}} \operatorname{rot} \underline{\mathbf{A}}\right)\right] & \equiv 0= \\
= & -\operatorname{div}[j \omega \gamma \underline{\mathbf{A}}]=\operatorname{div} \underline{\mathbf{J}}
\end{aligned}
$$

la seconde est une conséquence directe du couplage entre $\underline{\mathbf{A}}$ et $\underline{\psi}$ sur la surface extérieure $S_{13}$ [13]. En effet cette condition s'écrit :

$$
\mathbf{n} \cdot \underline{\mathbf{J}}=-\mathbf{n} \cdot j \omega \gamma \underline{\mathbf{A}}=\mathbf{n} \cdot \operatorname{rot}\left(\frac{1}{\mu_{\mathrm{e}}} \operatorname{rot} \underline{\mathbf{A}}\right) .
$$

Compte tenu de l'identité vectorielle :

$$
\operatorname{div}(\mathbf{U} \wedge \mathbf{V})=\mathbf{V} \cdot \operatorname{rot} \mathbf{U}-\mathbf{U} \cdot \operatorname{rot} \mathbf{V}
$$

la relation (11) devient :

$$
\begin{aligned}
\mathbf{n} \cdot \operatorname{rot} & \left(\frac{1}{\mu_{\mathrm{e}}} \operatorname{rot} \underline{\mathbf{A}}\right)= \\
= & \frac{1}{\mu_{\mathrm{e}}} \operatorname{rot} \underline{\mathbf{A}} \cdot \operatorname{rot} \mathbf{n}-\operatorname{div}\left[\mathbf{n} \wedge \frac{1}{\mu_{\mathrm{e}}} \operatorname{rot} \underline{\mathbf{A}}\right] .
\end{aligned}
$$

Comme rot $\mathbf{n} \equiv 0$, la composante normale de $\mathbf{J}$ à la surface $S_{13}$ devient :

$$
\mathbf{n} \cdot \underline{\mathbf{J}}=-\operatorname{div}\left[\mathbf{n} \wedge \frac{1}{\mu_{\mathrm{e}}} \operatorname{rot} \underline{\mathbf{A}}\right] \equiv-\operatorname{div}[\mathbf{n} \wedge \underline{\mathbf{H}}]
$$

soit, en tenant compte de la condition d'interface sur $S_{13}$ :

$$
\mathbf{n} \cdot \underline{\mathbf{J}}=-\operatorname{div}[\mathbf{n} \wedge \underline{\mathbf{H}}]=\operatorname{div}[\mathbf{n} \wedge \operatorname{grad} \underline{\psi}] .
$$

Le développement de (15) dans le système de coordonnées locales $\left(n, t_{1}, t_{2}\right)$, où $\mathbf{t}_{1}$ et $\mathbf{t}_{2}$ sont des tangentes à la surface $S_{13}$, donne :

$$
\begin{aligned}
& \mathbf{n} \cdot \underline{\mathbf{J}}=\operatorname{div}[ {[\mathbf{n} \wedge \operatorname{grad} \underline{\psi}]=} \\
&=\frac{\partial}{\partial t_{1}}\left(-\frac{\partial \underline{\psi}}{\partial t_{2}}\right)+\frac{\partial}{\partial t_{2}}\left(\frac{\partial \underline{\psi}}{\partial t_{1}}\right) \equiv 0 .
\end{aligned}
$$

\section{Forme variationnelle des équations.}

Pour établir une formulation variationnelle des équations (5), (6), (7), définissons au préalable leurs espaces fonctionnels $H^{1}\left(V_{1}\right)$ et $\left[H^{1}\left(V_{3}\right)\right]^{3}$ en les munissant des produits scalaires :

$$
\begin{aligned}
& (\underline{u} \cdot \underline{w})=\int_{V_{1}} \underline{u} \cdot \underline{w}^{*} \mathrm{~d} v \text { pour la zone } 1 \\
& (\underline{\mathbf{U}} \cdot \underline{\mathbf{W}})=\int_{V_{3}} \underline{\mathbf{U}} \cdot \underline{\mathbf{W}}^{*} \mathrm{~d} v \text { pour la zone } 3
\end{aligned}
$$

où $\underline{w}^{*}\left(\underline{\mathbf{W}}^{*}\right)$ désigne le conjugué de $\underline{w}(\underline{\mathbf{W}})$, et choisissons les fonctions tests $\underline{w}$ et $\underline{\mathbf{W}}$ dans la restriction des fonctions admissibles vérifiant les conditions aux limites essentielles sur $S_{1 \psi}, S_{3 \mathrm{~A}}$, $S_{3 \text { B }}$ et $S_{12}$. 
Après multiplication de (5) par $\underline{w}^{*}$, de (7) par $\underline{\mathbf{W}}^{*}$, et intégration par partie en faisant apparaître les conditions aux limites naturelles sur les surfaces $S_{1 \mathrm{q}}$ et $S_{13}$, on obtient, tous calculs faits :

$$
\begin{gathered}
R_{\psi}=-\int_{V_{1}} \mu_{1} \operatorname{grad} \underline{\psi} \cdot \operatorname{grad} \underline{w}^{*} \mathrm{~d} v-\int_{S_{13}} \operatorname{rot} \underline{\mathbf{A}} \cdot \mathbf{n}_{13} \underline{w}^{*} \mathrm{~d} s \equiv 0 \\
\forall \underline{w}^{*} \in\left[\begin{array}{c}
H^{1}\left(V_{1}\right) / \underline{w}=0 \operatorname{sur} S_{1 \psi} \\
\Delta \underline{w}(b)=\underline{w}\left(b_{2}\right)-\underline{w}\left(b_{1}\right) \equiv-\int_{a}^{b} \underline{\mathbf{J}}_{\mathrm{e}} \cdot \mathrm{dl} \\
\Delta \underline{w}(a)=0 \quad a, b \in S_{12}
\end{array}\right] \\
R_{\mathrm{A}}=\int_{V_{3}}\left\{\frac{1}{\mu_{\mathrm{e}}} \operatorname{rot} \underline{\mathbf{A}} \cdot \operatorname{rot} \underline{\mathbf{W}}^{*}+j \omega \gamma \underline{\mathbf{A}} \cdot \underline{\mathbf{W}}^{*}\right\} \mathrm{d} v+\int_{S_{13}}\left\{\mathbf{n}_{13} \wedge \operatorname{grad} \underline{\psi}\right\} \cdot \underline{\mathbf{W}}^{*} \mathrm{~d} s \equiv \mathbf{0} \\
\forall \underline{\mathbf{W}}^{*} \in\left[\begin{array}{r}
{\left[H^{1}\left(V_{3}\right)\right]^{3} / \underline{\mathbf{W}}=0 \operatorname{sur} S_{3 \mathrm{~A}}} \\
\underline{\mathbf{W}} \wedge \mathbf{n}=0 \operatorname{sur} S_{3 \mathrm{~B}}
\end{array}\right] .
\end{gathered}
$$

\section{Application au modèle pseudo-3D.}

Pour un actionneur tournant comportant un bobinage à $\mathrm{p}$ paires de pôles répartis le long de la direction circonférentielle $\theta$, les sources sont décrites par une nappe de courant de la forme :

$$
\underline{\mathbf{J}}_{\mathrm{e}}=\underline{\mathbf{J}}_{\mathrm{c}}(r, z) \mathrm{e}^{j(\omega t-p \theta)} .
$$

L'isotropie circonférentielle de structure autorise

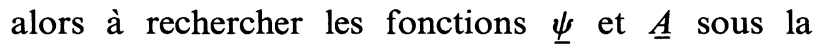
forme :

$$
\begin{aligned}
& \underline{\psi}(r, \theta, z)=\underline{\psi}_{\mathrm{c}}(r, z) \mathrm{e}^{-j p \theta} \\
& \underline{\mathbf{A}}(r, \theta, z)=\underline{\mathbf{A}}_{\mathrm{c}}(r, z) \mathrm{e}^{-j p \theta}
\end{aligned}
$$

(le cas du matériau saturable, pour lequel l'isotropie circonférentielle est a priori détruite, sera discuté ultérieurement). Introduisons les notations suivantes

$$
\begin{aligned}
\operatorname{grad} \underline{\psi} & =\operatorname{grad}_{\mathrm{c}} \underline{\psi}_{\mathrm{c}} \mathrm{e}^{-j p \theta} \\
\operatorname{rot} \underline{\mathbf{A}} & =\operatorname{rot}_{\mathrm{c}} \underline{\mathbf{A}}_{\mathrm{c}} \mathrm{e}^{-j p \theta}
\end{aligned}
$$

en définissant les opérateurs complexes $\operatorname{grad}_{c}$ et rot $_{\mathrm{c}}$ comme :

$$
\begin{gathered}
\operatorname{grad}_{\mathrm{c}} \underline{\psi}_{\mathrm{c}}=\left[\begin{array}{c}
\frac{\partial \underline{\psi}_{\mathrm{c}}}{\partial r} \\
-\frac{j \underline{p}}{r} \underline{\psi}_{\mathrm{c}} \\
\frac{\partial \underline{\psi}_{\mathrm{c}}}{\partial z}
\end{array}\right] ; \\
\operatorname{rot}_{\mathrm{c}} \underline{\mathbf{A}} \mathrm{c}=\left[\begin{array}{c}
-\frac{j \underline{p}}{r} \underline{A}_{\mathrm{c} z}-\frac{\partial \underline{A}_{\mathrm{c} \theta}}{\partial r} \\
\frac{\partial \underline{A}_{\mathrm{c} r}}{\partial z}-\frac{\partial \underline{A}_{\mathrm{c} z}}{\partial r} \\
\frac{\partial\left(r \underline{A}_{\mathrm{c} \theta}\right)}{\partial r}+\frac{j \underline{p}}{r} \underline{A}_{\mathrm{cr} r}
\end{array}\right] .
\end{gathered}
$$

L'intégration des équations variationnelles (19) et (20) suivant la coordonnée $\theta$ permet alors de ramener à deux la dimension de l'espace géométrique :

$$
\begin{aligned}
& R_{\psi}=-\quad \mu_{1} \operatorname{grad}_{\mathrm{c}} \underline{\psi}_{\mathrm{c}} \cdot \operatorname{grad}_{\mathrm{c}}^{*} \underline{w}^{*} r \mathrm{~d} r \mathrm{~d} z-\int_{L} \operatorname{rot}_{\mathrm{c}} \underline{\mathbf{A}}_{\mathrm{c}} \cdot \mathbf{n}_{13} \underline{w}^{*} r \mathrm{~d} \boldsymbol{\ell} \equiv 0 \\
& R_{\mathrm{A}}=\int_{\Sigma_{3}}\left\{\frac{1}{\mu_{\mathrm{e}}} \operatorname{rot}_{\mathrm{c}} \underline{\mathbf{A}}_{\mathrm{c}} \cdot \operatorname{rot}_{\mathrm{c}}^{*} \underline{\mathbf{W}}^{*}+j \omega \gamma \underline{\mathbf{A}}_{\mathrm{c}} \cdot \underline{\mathbf{W}}^{*}\right\} r \mathrm{~d} r \mathrm{~d} z+\int_{L_{13}}\left\{\mathbf{n}_{13} \wedge \operatorname{grad}_{\mathrm{c}} \underline{\psi}_{\mathrm{c}}\right\} \cdot \underline{\mathbf{W}} * r \mathrm{~d} \boldsymbol{\ell} \equiv \mathbf{0}
\end{aligned}
$$

avec $\Sigma_{1}, \Sigma_{3}$ et $L_{13}$ les traces de $V_{1}, V_{3}$ et $S_{13}$ dans le plan $r-z$.

\section{5. forme discrétisée des équations.}

L'écriture des opérateurs vectoriels en coordonnées cylindriques introduit des termes en $1 / r$ gênants du point de vue numérique. Pour s'en affranchir, et sans trop élever le degré des intégrants, introduisons les variables intermédiaires $\underline{\mathbf{V}}$ et $\underline{u}$ définies comme suit :

$$
\underline{\mathbf{A}}_{\mathrm{c}}=\sqrt{r} \underline{\mathbf{V}}(r, z) ; \quad \underline{\psi}_{\mathrm{c}}=\sqrt{r} u(r, z) .
$$


La méthode des éléments finis conduit alors à rechercher une approximation de $\underline{\mathbf{V}}$ et $\underline{u}$ sous la forme :

$$
\begin{aligned}
\underline{\mathbf{V}}(r, z) & =\sum_{i=1}^{\mathrm{NNT}} \underline{\mathbf{V}}^{i} N_{i}(r, z) ; \\
\underline{u}(r, z) & =\sum_{i=1}^{\mathrm{NNT}} \underline{u}^{i} N_{i}(r, z) .
\end{aligned}
$$

Utilisons la méthode de Galerkine pour introduire les fonctions tests $\underline{\mathbf{W}}^{*}$ et $\underline{w}^{*}$ sous la forme :

$$
\begin{aligned}
& \underline{\mathbf{W}}^{*} \equiv \delta \underline{\mathbf{V}}^{*}= \sum_{i=1}^{\mathrm{NNT}} \delta \underline{\mathbf{V}}^{i} N_{i}(r, z) ; \\
& \underline{w}^{*} \equiv \delta \underline{u}=\sum_{i=1}^{\mathrm{NNT}} \delta \underline{u}^{i} N_{i}(r, z)
\end{aligned}
$$

et écrire les résidus $R_{\mathrm{A}}$ et $R_{\psi}$ sous forme matricielle :

$$
\begin{aligned}
R=\left\langle\delta \underline{Y}^{*}\right\rangle\{[\mathrm{KC}]\{\underline{Y}\}-\{\underline{\mathrm{FC}}\}\} & \equiv 0 \\
& \forall\left\langle\delta \underline{Y}^{*}\right\rangle
\end{aligned}
$$

où le vecteur ligne $\langle\underline{Y}\rangle$ désigne l'ensemble des inconnues nodales $\left(\underline{u}^{i}, \underline{V}_{r}^{i}, \underline{V}_{\theta}^{i}, \underline{V}_{z}^{i}\right)$ attachées aux nœuds $i=1, \ldots$, NNT de la discrétisation.

L'expression de $[\mathrm{KC}]$ est détaillée dans [7], ainsi que la méthodologie utilisée pour introduire les termes de couplage entre les degrés de liberté $\underline{u}^{i}$ et $\left(\underline{V}_{r}^{i} ; \underline{V}_{\theta}^{i} ; \underline{V}_{z}^{i}\right)$ attachés aux nœuds de la frontière $S_{13}$.

On remarquera qu'à chaque nœud de discrétisation sont attachés trois degrés de liberté complexes dans la zone 3 , un dans la zone 1 , et quatre sur la surface $S_{13}$. Un degré de liberté complexe correspondant à deux degrés de liberté réels, et avec une utilisation systématique de réels double précision, on peut déjà préjuger de la taille des problèmes traités, et entrevoir l'intérêt d'éviter si possible une modélisation géométrique 3D.

\section{Modèle pseudo-3D non linéaire.}

La saturation magnétique a théoriquement deux conséquences :

- d'une part la solution contient des harmoniques temporelles, même avec des sources sinusoïdales

- d'autre part l'isotropie circonférentielle est détruite, car tous les points situés dans un même plan $z=$ Cste n'étant pas magnétisée au même instant par le même champ, la perméabilité $\mu$ devient une fonction de $\theta$.

Elle exige donc en toute rigueur la résolution temporelle pas à pas d'un modèle 3D. Or il est toujours très coûteux d'étudier un régime stationnaire en traitant la dimension temps par une méthode pas à pas, et un considérable gain de calcul est réalisé quand on travaille dans le domaine en fréquence. L'expérience des modèles $2 \mathrm{D}$ a montré à cet effet que l'on pouvait adapter le problème non linéaire en utilisant la notion de perméabilité magnétique équivalente: chaque point du matériau est caractérisé par une perméabilité magnétique ne dépendant que de la valeur de l'amplitude de l'induction en ce point, induction supposée rester à variations temporelles sinusoïdales.

On peut définir $\mu_{\mathrm{e}}$ de différentes façons [14]. Des expériences numériques sur des modèles $1 \mathrm{D}$ et $2 \mathrm{D}$ du fer massif nous ont toutefois conduits à retenir la définition la plus simple et à la transposer au modèle pseudo-3D comme suit [7] :

$$
\left[\begin{array}{l}
\mu_{\mathrm{e}}=\mu\left(B_{\mathrm{e}}\right) \\
B_{\mathrm{e}}^{2}=\left|B_{r}\right|^{2}+\left|B_{\theta}\right|^{2}+\left|B_{z}\right|^{2} \equiv|\operatorname{rot} \underline{\mathbf{A}}|^{2} \equiv\left|\operatorname{rot}_{\mathrm{c}} \underline{\mathbf{A}}_{\mathrm{c}}\right|^{2} \\
\underline{\mathbf{B}}_{\mathrm{c}}=\operatorname{rot}_{\mathrm{c}} \underline{\mathbf{A}}_{\mathrm{c}} \equiv \mu_{\mathrm{e}} \underline{\mathbf{H}}_{\mathrm{c}} \\
\mu=\operatorname{caractéristique~statique~d'aimantation~moyenne~}_{\boldsymbol{\mu}}=\left\langle\underline{A}_{\mathrm{c}}, \underline{A}_{\mathrm{c} \theta}, \underline{A}_{\mathrm{c} z}\right\rangle=\text { amplitude complexe de } \boldsymbol{A}
\end{array}\right.
$$

Remarquons que cette définition de $\mu_{\mathrm{e}}$ est cohérente avec l'hypothèse d'isotropie circonférentielle inhérente au modèle pseudo-3D : la restriction (23) du champ de vecteurs admissibles permet d'affirmer que des éléments de matériau de coordonnées $(r, \theta, z)$ et $(r, \theta+\Delta \theta, z)$ sont soumis à des inductions de même amplitude $B_{\mathrm{e}}$ et, d'après la définition (33), sont caractérisés par la même valeur de perméabilité équivalente $\mu_{\mathrm{e}}$.

La résolution de (32), compte tenu de la loi de milieu (33), est basée sur une méthode de Newton-
Raphson incrémentale, nécessaire étant donné le haut niveau de saturation existant dans le fer massif conducteur. Un développement en série du résidu $R$ de (32) permet de passer d'une approximation $\left\{\underline{Y}^{p}\right\}$ à une approximation $\left\{\underline{Y}^{p+1}\right\}$ par :

$$
\begin{aligned}
& \left\{\underline{Y}^{p+1}\right\}=\left\{\underline{Y}^{p}\right\}+\{\Delta \underline{Y}\} \\
& {\left[\operatorname{KCT}\left(\left\{\underline{Y}^{p}\right\}\right)\right]\{\Delta \underline{Y}\}-\left\{\operatorname{RC}\left(\left\{\underline{Y}^{p}\right\}\right)\right\} \equiv 0 .}
\end{aligned}
$$

Pour définir correctement la matrice tangente [KCT], il faut calculer des expressions de la forme 
$\partial \nu_{\mathrm{e}} / \partial \underline{B}_{\mathrm{c} i}, i=r, \theta, z$. Avec la définition (33) de $\nu_{\mathrm{e}}$, la méthodologie utilisée en magnétostatique dans [15] peut s'étendre sans difficulté au cas du problème pseudo-3D traité avec la notation complexe, ce qui permet d'exprimer la matrice tangente exactement comme suit [7] :

$$
\begin{aligned}
{[\mathrm{KCT}]=} & {[\mathrm{KC}]+[\mathrm{KNL}] } \\
{[\mathrm{KNL}]=} & \sum_{k=1}^{\mathrm{NNT}} \sum_{\ell=1}^{\mathrm{NNT}}\left[\mathrm{KNL}^{k \ell}\right] \\
{\left[\mathrm{KNL}^{k \ell}\right]=} & \int_{\Sigma_{3}} 2 \frac{\partial \nu_{\mathrm{e}}}{\partial\left|\underline{B}_{\mathrm{c}}\right|^{2}}\left\{\operatorname{grad}_{\mathrm{c}}^{*} N_{k} \wedge \underline{\mathbf{B}}_{\mathrm{c}}\right\} \times \\
& \times\left\langle\operatorname{grad}_{\mathrm{c}} N_{\ell} \wedge \underline{\mathbf{B}}_{\mathrm{c}}^{*}\right\rangle r^{2} \mathrm{~d} r \mathrm{~d} z .
\end{aligned}
$$

\section{Description des sources.}

La mise en œuvre du modèle pseudo-3D passe par la description des sources sous forme de nappes de courant à divergence nulle. Celles-ci s'introduisent naturellement par l'intermédiaire du potentiel vecteur électrique $\underline{\mathbf{T}}$ tel que $\underline{\mathbf{J}}_{\mathrm{e}}=\operatorname{rot} \underline{\mathbf{T}}$ [16], qui, dans le système de coordonnées locales $(u, \theta, v)$ attaché à la nappe de courant, peut être identifié comme :

$$
\underline{\mathbf{T}}=\mid \begin{aligned}
& \underline{T}_{u}(u, \theta, v)=\underline{T}_{1}(u) \underline{T}_{2}(v) \mathrm{e}^{j(\omega t-p \theta)} \\
& \underline{T}_{\theta}(u, \theta, v)=0 \\
& \underline{T}_{v}(u, \theta, v)=0
\end{aligned}
$$

Dans le cas de la nappe de courant dimensionnée pour créer uniquement un champ tournant, la fonction $\underline{T}_{2}(v)$ peut être choisie égale à l'unité le long de l'empilage des tôles statoriques $(u \equiv r$, $\left.v \equiv z=0 \ldots z_{1}\right)$, pour décroître vers zéro le long des têtes de bobines localisées entre les coordonnées $z_{1}$ et $z_{2}$. Pour simplifier, on pourra supposer cette décroissance linéaire, étant entendu que dans le cas d'une étude spécifique, cette fonction pourra être plus élaborée $[16,20]$. La fonction $\underline{T}_{1}(u)$ est alors définie sans ambiguïté. Dans le cas simplifié d'une nappe de courant d'amplitude $J_{\mathrm{m}}$, localisée sur un cylindre de rayon $R_{\mathrm{s}}$ et d'axe $\mathrm{O} z$, avec des têtes de bobines comprises entre les coordonnées $z=z_{1}$ et $z=z_{2}$, on est conduit à décrire la densité de courant équivalente $\underline{\mathbf{J}}_{\mathrm{e}}$ comme :

$$
\begin{gathered}
\underline{J}_{r}=0 \\
\underline{\mathrm{e}}-\quad- \\
\underline{J}_{z}=J_{\mathrm{m}} \mathrm{e}^{j(\omega t-p \theta)} ; \\
\text { pour } \quad 0 \leqslant z \leqslant z_{1} \\
=\mid \begin{array}{l}
\underline{J}_{r}=0 \\
\underline{J}_{\theta}=\frac{j R_{\mathrm{s}}}{z_{2}-z_{1}} \frac{J_{\mathrm{m}}}{p} \mathrm{e}^{j(\omega t-p \theta)} \\
\underline{J}_{z}=\frac{z-z_{2}}{z_{1}-z_{2}} J_{\mathrm{m}} \mathrm{e}^{j(\omega t-p \theta)} \\
z_{1} \leqslant z \leqslant z_{2} ;
\end{array}
\end{gathered}
$$

Compte tenu des conditions aux limites essentielles (19) à imposer sur la frontière $L_{12}$, il faut introduire numériquement ces sources sous la forme d'une coupure sur le potentiel scalaire $\psi$. Cette dernière peut s'interpréter comme une relation linéaire entre deux degrés de liberté, dont la prise en compte constitue une étape standard de programmation en éléments finis [17].

\section{Validité du modèle pseudo-3D.}

La validation du modèle présenté comporte deux aspects :

- validation numérique pour tester les algorithmes

- validation physique pour confirmer les hypothèses simplificatrices.

VALIDITÉ NUMÉRIQUE DU MODÈLE. - On étudie un moteur asynchrone à rotor lisse dont on néglige les effets de longueur finie, ce qui revient à supposer le moteur de longueur infinie. Ceci permet de comparer les résultats obtenus avec un modèle $2 \mathrm{D}$, dont la validité n'est plus à démontrer, et le modèle pseudo-3D, pour lequel il suffit d'imposer des conditions de périodicité pour simuler la longueur infinie (on ne dispose pas de référence analytique quand il s'agit de traiter simultanément de la saturation et des anisotropies axiales).

Les données numériques sont les suivantes :

Rotor :

- acier 35CD4 avec caractéristique moyenne d'aimantation approchée par la courbe :

$$
\begin{gathered}
\nu_{r}(B)=\frac{1}{477}+\left(0,7-\frac{1}{477}\right) \frac{B^{8}}{13000+B^{8}} . \\
\mu=\frac{\mu_{0}}{\nu_{r}}
\end{gathered}
$$

avec

- conductivité $\gamma=3,42 \times 10^{6} \Omega^{-1} \mathrm{~m}^{-1}$ à $150^{\circ} \mathrm{C}$

- diamètre $=234 \mathrm{~mm}$

- longueur utile $=460 \mathrm{~mm}$

- vitesse $=0 \mathrm{t} / \mathrm{s}$.

Entrefer corrigé $=3,8 \mathrm{~mm}$.

Bobinage statorique :

- bobinage sinusoïdal équivalent : $n(\theta)=$ $52,7 \cos \theta$ spires/radian

- densité de courant $J_{\mathrm{m}}=27667 \mathrm{~A} / \mathrm{m}$; fréquence $f_{\mathrm{s}}=1 \mathrm{~Hz}$.

Fréquence de glissement rotorique : $f_{\mathrm{r}}=1 \mathrm{~Hz}$.

La discrétisation est réalisée avec des éléments quadrilatéraux cubiques à 12 nœuds. Les résultats de calcul, ramenés à la longueur utile de rotor, sont regroupés dans le tableau de la figure 2 (cf. le point de fonctionnement à $1 \mathrm{~Hz}$ de la courbe $\mathrm{n}^{\circ} 4$ des Figs. 8, 9, 10). Ils confirment la validité numérique du modèle pseudo-3D, les écarts avec le modèle $2 \mathrm{D}$ 


\begin{tabular}{|c|c|c|c|c|c|}
\hline 1 & 1 & Modèle 20 & & èle Pseud & \\
\hline I Couple ( $\mathrm{mN})$ & 1 & 271,57 & 1 & 270,64 & । \\
\hline I Pertes Joules (W) & 1 & $1706: 4$ & 1 & 1700,4 & 1 \\
\hline $\mid \cos \varphi$ & 1 & 0,6067 & 1 & 0,6123 & 1 \\
\hline I Tension induite (V) & 1 & 2,6785 & 1 & 2,6447 & 1 \\
\hline I Induction $\quad \mid B_{r}$ & 1 & 0,551 & 1 & 0,551 & । \\
\hline | entrefer (T) | $\mathrm{B}_{\theta}$ & 1 & 0,043 & 1 & 0,043 & 1 \\
\hline $1 \quad 1 B_{2}$ & 1 & - & 1 & $10^{-16}$ & 1 \\
\hline I induction $\quad \mid \mathrm{Br}_{r}$ & 1 & 0.558 & 1 & 0.558 & 1 \\
\hline Irotor (T) $\mid E_{\theta}$ & 1 & 1,98 & 1 & 1,99 & 1 \\
\hline $1 \quad 1 B_{2}$ & 1 & - & 1 & $10^{-15}$ & । \\
\hline
\end{tabular}

Fig. 2. - Test : comparaison des modèles $2 \mathrm{D}$ et pseudo-3D (fréquence statorique $1 \mathrm{~Hz}$ - rotor à l'arrêt).

[Test : comparison of $2 \mathrm{D}$ and quasi-3D models (stator frequency : $1 \mathrm{~Hz}$ - locked rotor.]

restant inférieurs à $5 \%$, autant sur les grandeurs locales comme l'induction, que sur toutes les grandeurs énergétiques (il faut noter que ces grandeurs se réfèrent à tout le moteur, pour une fréquence d'alimentation de $1 \mathrm{~Hz}$, égale à la fréquence de glissement avec le rotor à l'arrêt). On peut attribuer ce faible écart d'une part au fait que le modèle pseudo-3D travaille sur deux types d'inconnues, $\underline{\mathbf{A}}_{\mathrm{c}}$ et $\underline{\psi}_{\mathrm{c}}$ (une seule inconnue pour le modèle $2 \mathrm{D}$ : $\underline{A}_{z}$ ), et que d'autre part le champ de variations admissibles de ces inconnues est légèrement supérieur à celui du modèle $2 \mathrm{D}$ par suite du changement de variable (29).

Notons que dans ce tableau, les valeurs du couple sont obtenues avec la méthode du tenseur de Maxwell, la tension induite et le $\cos \psi$ du bobinage statorique étant calculés à partir des énergies actives et réactives, et du courant.

VALIDITÉ PHYSIQUE DU MODÈLE. - Rappelons que le modèle pseudo-3D non linéaire repose sur deux hypothèses simplificatrices fondamentales :

- l'isotropie circonférentielle de structure

- la définition de la perméabilité magnétique équivalente.

La notion de perméabilité magnétique équivalente a déjà été utilisée par de nombreux auteurs dans des modèles $2 \mathrm{D}[14,21,22]$. Pour son extension (33) à la formulation pseudo-3D, nous avons retenu le modèle le plus simple possible. On peut justifier ce choix en se référant à des études $2 \mathrm{D}$ effectuées en régime stationnaire, lesquelles n'ont révélé aucune différence significative avec un calcul pas à pas exact [7]. Ces conclusions ont aussi été confirmées par l'expérimentation sur un moteur asynchrone à rotor massif $1 \mathrm{MW}-20000 \mathrm{t} / \mathrm{min}$, avec laquelle aucun écart énergétique supérieur à $15 \%$ n'a pu être relevé, ce qui constitue un excellent résultat compte tenu de tous les phénomènes secondaires non inclus dans le modèle (échauffement du rotor, pertes de surface, pertes hydrauliques, alimentation par commutateur de courant, etc.). Il faut également souligner, à la décharge du modèle simpliste retenu pour définir $\mu_{\mathrm{e}}$, que la caractéristique magnétique des aciers utilisés est, au contraire de celles des tôles, souvent mal connue, avec en outre un cycle d'hystérésis assez conséquent.

Quant à la première hypothèse, on ne redémontrera pas ici la validité de la notion de "coefficient de Carter », permettant de remplacer la structure encochée du stator par un entrefer lisse équivalent. Le seul point délicat peut être lié à l'apparition des pertes supplémentaires de surface dues à la rotation à grande vitesse du rotor devant la structure encochée du stator. Mais moyennant une construction appropriée du rotor, celles-ci sont suffisamment minimisées pour justifier le processus d'homogénéisation du stator dans la direction circonférentielle. On a par ailleurs montré qu'une utilisation judicieuse du modèle pseudo-3D permettait de modéliser ces pertes dans un rotor rainuré circonférentiellement [7]. 
Enfin la cohérence des résultats présentés dans le prochain paragraphe confortera la validité physique du modèle proposé, d'autant plus qu'ils recoupent les calculs 2D développés avec les facteurs correctifs habituellement retenus.

\section{Application : effets d'extrémité dans moteur asyn- chrone à rotor massif.}

Des études analytiques ont montré que les effets de longueur finie pouvaient détériorer significativement les performances des moteurs à rotor massif, au regard des prédéterminations obtenues à partir de modèles supposés arbitrairement longs. Car en réalité les courants induits présentent, en plus de leur composante axiale utile, des composantes tangentielle et radiale qui correspondent à la fermeture des lignes de courant tout le long du rotor.

Dans le cas d'un moteur à rotor ferromagnétique massif, il est très difficile de prendre simultanément en compte la longueur finie et la saturation par voies analytiques. En fait ces dernières séparent les deux phénomènes, l'effet de longueur finie ne faisant que corriger les résultats du modèle de longueur infinie. Il existe à ce sujet deux méthodes antagonistes qui supposent :

- soit le rotor saturé uniquement dans sa région centrale, avec ses zones frontales non saturées [18]

- soit le rotor saturé uniformément sur toute sa longueur, y compris dans les zones frontales [19].

Le modèle numérique pseudo-3D permet de donner un élément de réponse supplémentaire à ce problème de longueur finie, car il tient compte de la saturation en incluant toutes les anisotropies axiales de structure.

On utilise ce modèle pour déterminer les performances du moteur représenté en coupe axiale sur la figure 3. Les données numériques sont celles utilisées dans le problème test du paragraphe 8 , l'amplitude de l'excitation étant conservée à $J_{\mathrm{m}}=27667 \mathrm{~A} / \mathrm{m}$.

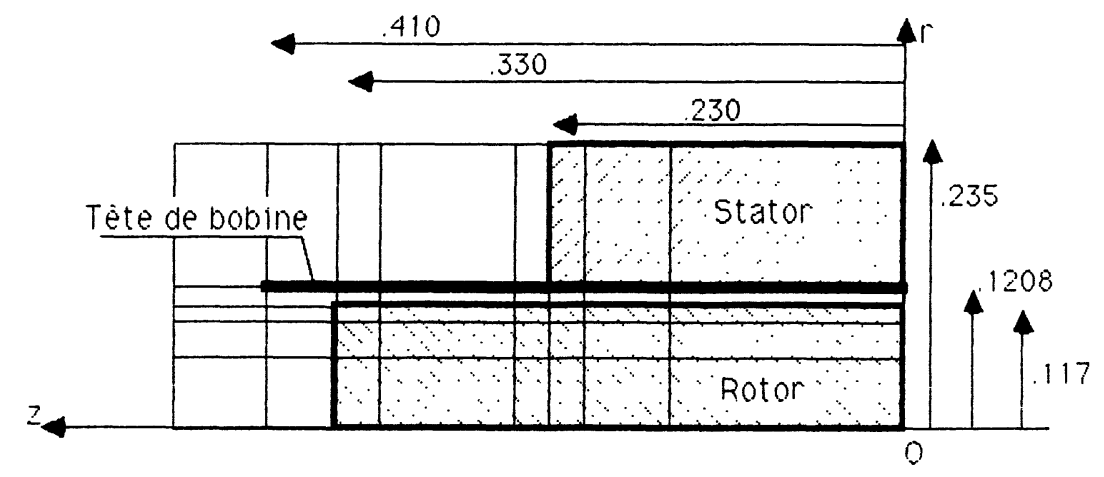

Fig. 3. - Moteur à rotor massif : modèle pseudo-3D. Discrétisation avec des rectangles Q12.

[Solid-rotor induction machine : quasi-3D model. Mesh with cubic quadrilateral elements (Q12).]

9.1 Distribution DU CHAMP ÉLECTROMAGNÉTIQUE. - Examinons tout d'abord comment les effets d'extrémité viennent perturber la distribution du champ électromagnétique.

Les figures 4 et 5 donnent la répartition de l'amplitude des composantes radiale, tangentielle, et axiale

, ction ma néti ue de art et d'autre de la surface séparant le rotor et l'entrefer, pour deux valeurs de la fréquence de glissement rotorique : 1 et $10 \mathrm{~Hz}$.

Les figures 6 et 7 donnent les distributions correspondantes de l'amplitude des composantes du vecteur densité de courant induit.

On constate qu'au niveau de l'entrefer l'induction reste principalement radiale. Mais la fermeture des lignes de courant en $\theta$ se traduit par une augmentation progressive de cette composante, du milieu vers l'extrémité du stator. Dans le cas présent cette variation est dans un rapport 2 et semble indépen- dante du niveau de flux dans l'entrefer (le rotor est pratiquement toujours saturé, du moins en surface).

Dans la couche périphérique du rotor, le champ magnétique est redistribué presque tangentiellement. Là encore la composante tangentielle $\boldsymbol{B}_{\theta}$ diminue sensiblement en se rapprochant de l'extrémité du stator, au profit de la composante $B_{z}$. Mais le niveau de saturation du rotor reste constant tout e long de sa surface, même dans la région frontale située à l'extérieur du stator, où pratiquement aucun flux ne sort de sa surface, l'induction radiale y étant très faible. Cette remarque, valable à toute fréquence, semble contredire les hypothèses formulées dans [18], et confirmer celles de [9] qui supposent le rotor dans un état de saturation uniforme. On retrouve aussi le fait que la composante radiale $B_{r}$ a toujours, dans la partie utile du rotor, une valeur d'autant moins négligeable que la fréquence est faible. 


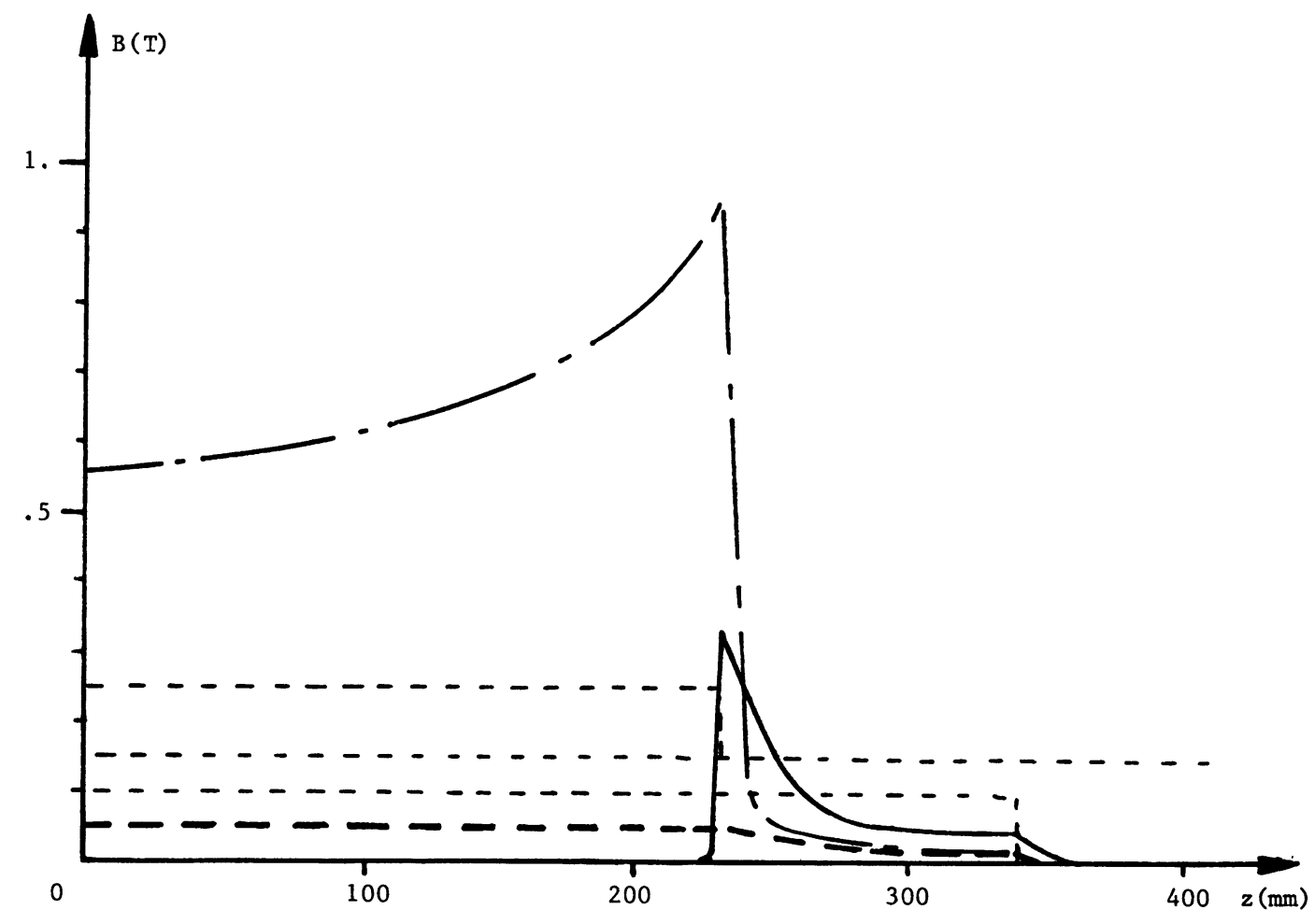

(1)

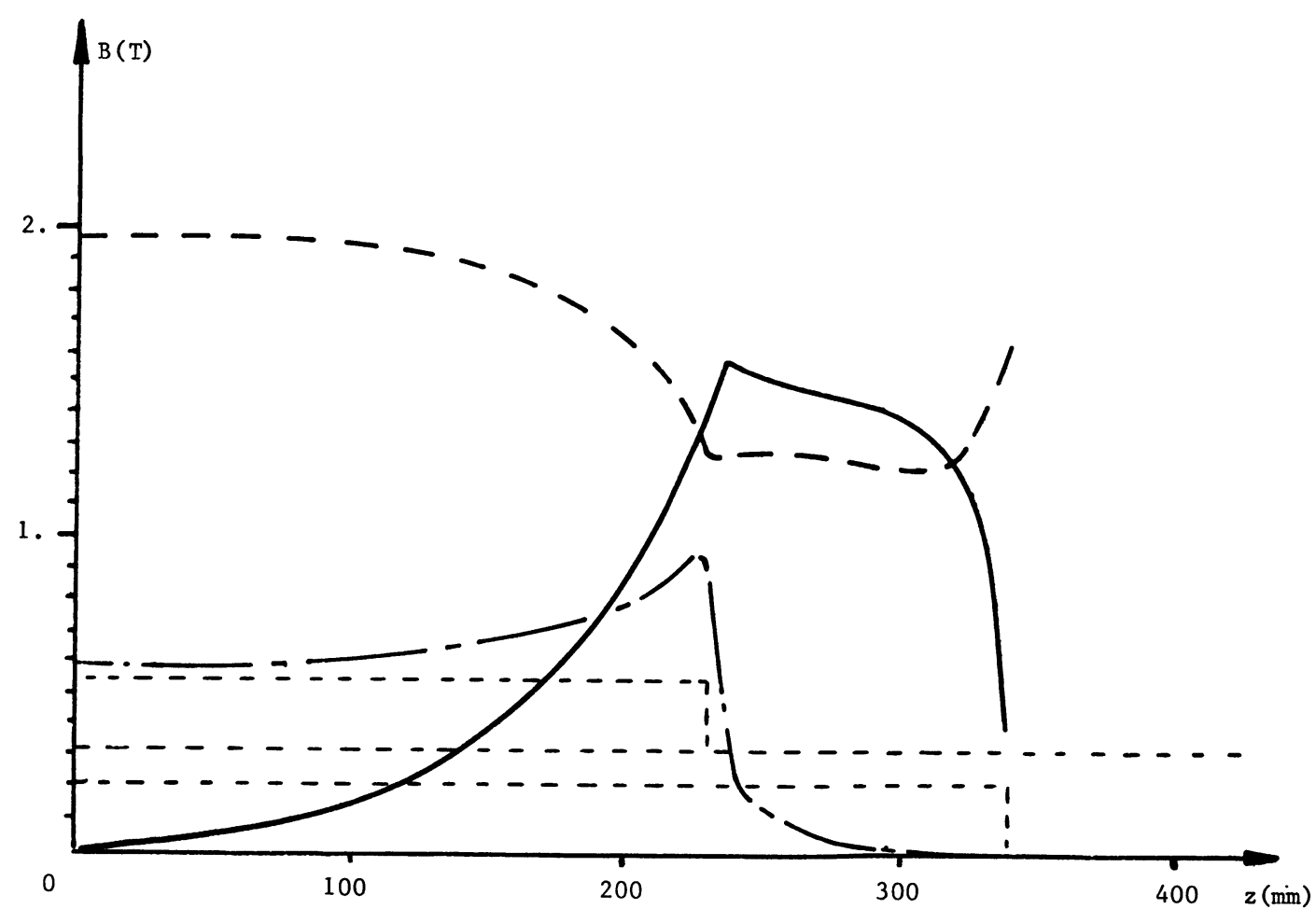
Fig. 4. Effets de longueur finie : induction pour $f_{\mathrm{r}}=1 \mathrm{~Hz} .(--) B_{1} ;(---) B_{\theta} ;(-117,2 \mathrm{~mm}$. b) Rotor: $r=116,8 \mathrm{~mm}$.
$r=11$

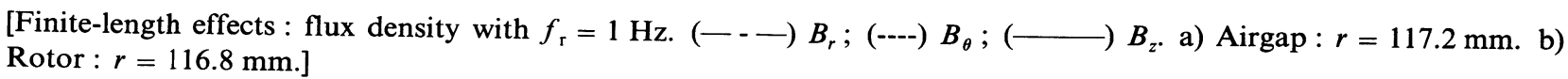




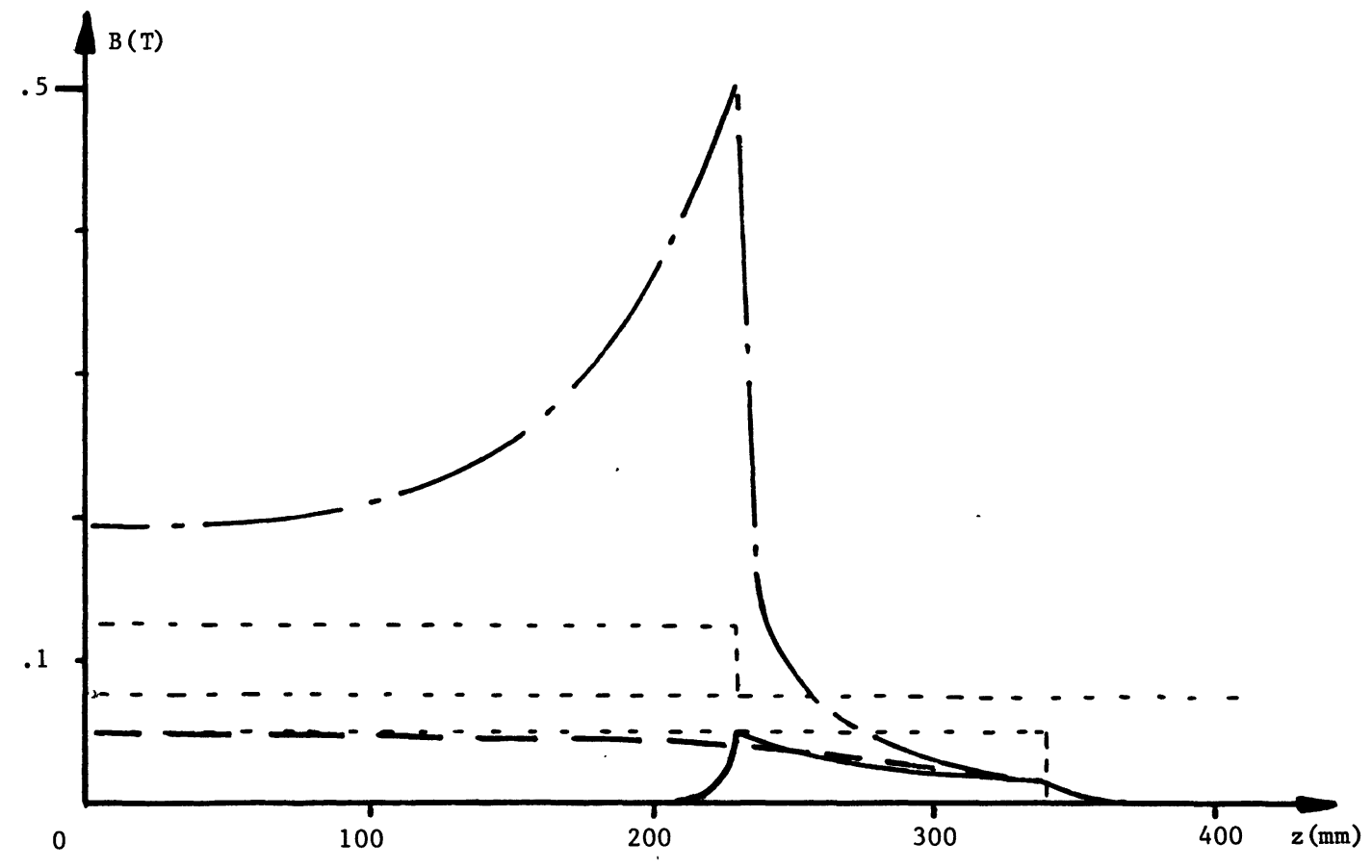

(1)

3.
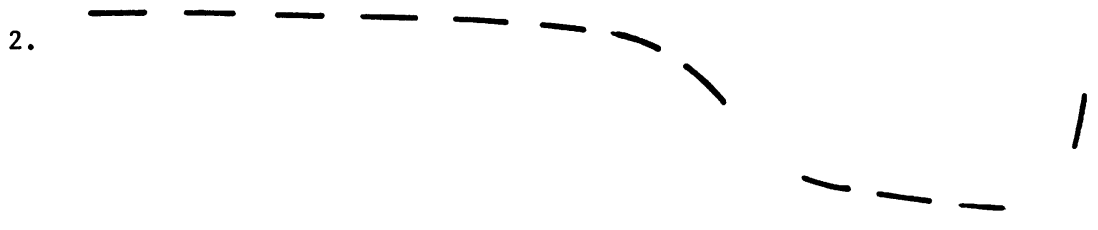

1.

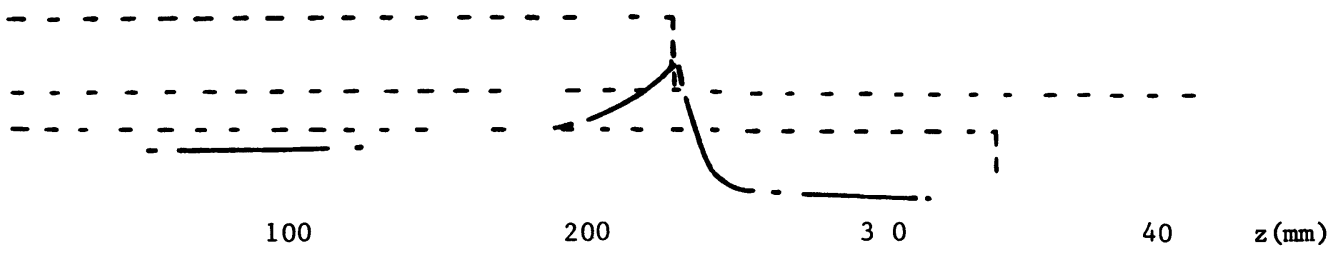

Fig. 5. - Effets de longueur finie : induction pour $f_{\mathrm{r}}=10 \mathrm{~Hz}$. (- - $B_{r} ;(---) B_{\theta} ;\left(-\right.$ - $B_{z}$. a) Entrefer : $r=117,2 \mathrm{~mm}$. b) Rotor : $r=116,8 \mathrm{~mm}$.

[Finite-length effects : flux density with $f_{\mathrm{r}}=10 \mathrm{~Hz}$. (- - $B_{r} ;(---) B_{\theta}$; ( - $B_{z}$. a) Airgrap : $r=117.2 \mathrm{~mm}$. b) Rotor : $r=116.8 \mathrm{~mm}$.] 
Des conclusions semblables peuvent être déduites pour la distribution des courants induits. La composante axiale $J_{z}$, maximale au centre du rotor, diminue vers les extrémités au profit de la composante tangentielle $J_{\theta}$. Quant à la composante radiale $J_{r}$, elle ne devient significative qu'au voisinage des faces frontales, assurant avec $J_{\theta}$ une divergence nulle à la densité de courant.

\subsection{Couple ÉlectromagnétiQue - FACTEur} DE PUISSANCE. - On compare les résultats obtenus avec le modèle pseudo-3D et le modèle $2 \mathrm{D}$. Pour ce dernier, les calculs sont faits successivement sans tenir compte des facteurs correctifs de longueur finie, puis en modifiant la conductivité $\gamma$ du rotor comme indiqué dans [19] (avec les données numériques utilisées : $\left.\gamma_{\text {eff }}=\gamma \times 0,55\right)$.

La figure 8 donne les caractéristiques couple/fréquence de glissement obtenues en maintenant le courant statorique à une valeur constante. On constate que les effets d'extrémité sont correctement intégrés dans le modèle $2 \mathrm{D}$ corrigé, alors que le même modèle $2 \mathrm{D}$ non corrigé conduit à des écarts significatifs. Cependant, pour des fréquences situées en deçà de la fréquence du couple maximal, cette correction du modèle 2D n'est plus suffisante (cf. l'agrandissement de la Fig. 9).
La figure 10 rapporte les variations correspondantes du facteur de puissance. Pour cette grandeur, le modèle $2 \mathrm{D}$ même corrigé conduit déjà à des valeurs par excès.

9.3 INFLUENCE DE PARAMÈTRES SPÉCIFIQUES A LA LONGUEUR FINIE. - Deux paramètres spécifiques à la longueur finie sont difficilement pris en compte par l'approche analytique: la forme et l'extension axiale du rotor en dehors du stator, et la fermeture des courants sources par les têtes de bobines.

Par exemple l'étude simplifiée développée dans [19] arrive à la conclusion que la forme et la longueur de la partie frontale du rotor située à l'extérieur du stator n'ont aucune influence sur la création de couple, pour autant que la topologie frontale soit continue à la traversée de l'axe du rotor. Or la courbe 2 reportée sur la figure 8 , calculée en supposant la longueur du rotor égale à celle $\mathrm{du}$ stator, indique une diminution sensible du couple.

De même, si on conserve la longueur initiale de rotor, tout en raccourcissant à l'extrême les têtes de bobines (une faible longueur est nécessaire pour assurer $\operatorname{div} \underline{\mathbf{J}}_{\mathrm{e}}=0$ ), on retrouve une diminution semblable de couple (courbe 3 de la Fig. 8).

On arrive ainsi à la conclusion logique que l'inte-

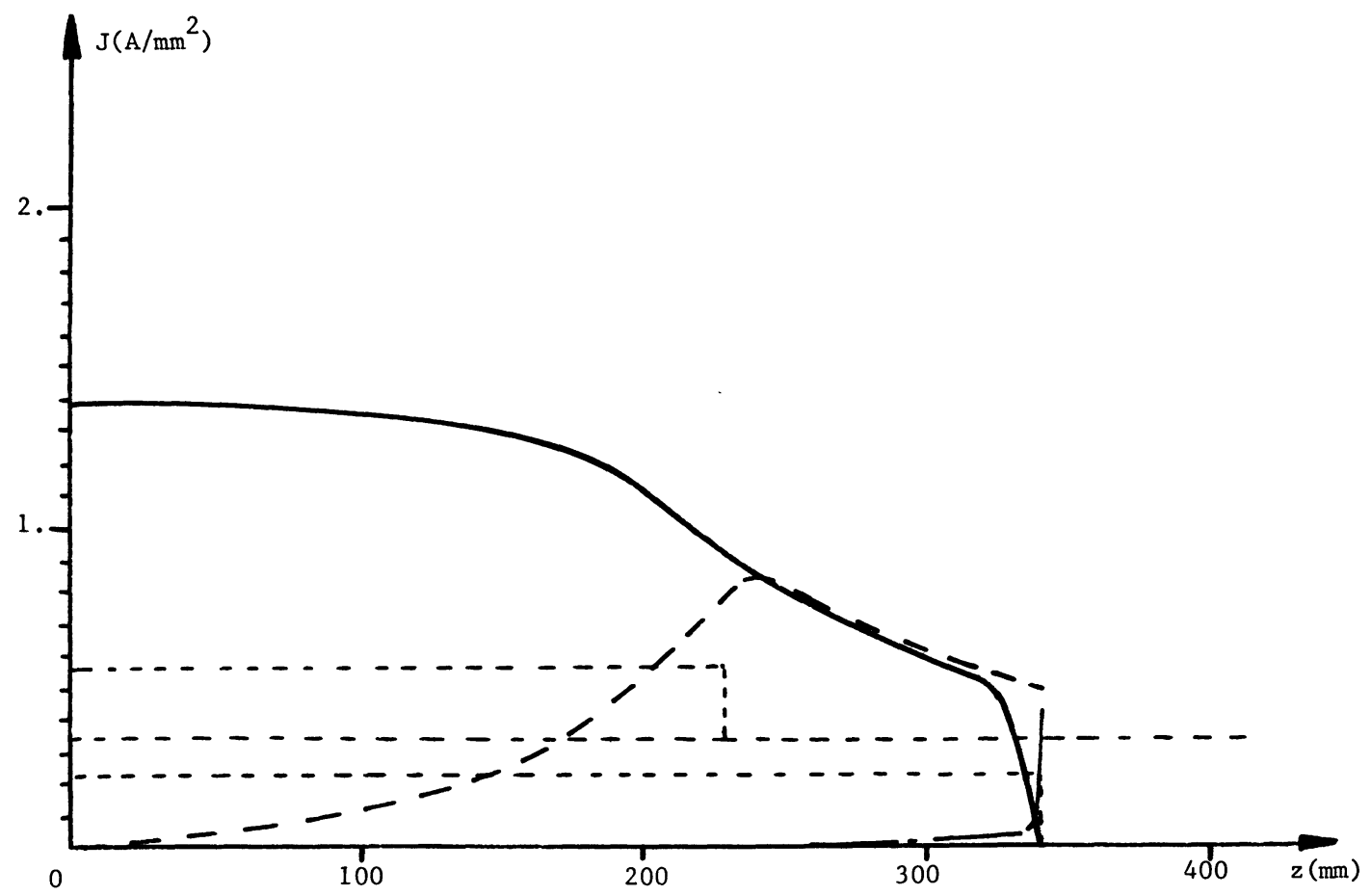

Fig. 6. - Effets de longueur finie : courants induits pour $f_{\mathrm{r}}=1 \mathrm{~Hz}$. (-- - $J_{r} ;(---) J_{\theta} ;\left(\longrightarrow\right.$ (一) $J_{z}$ a) $r=116,8 \mathrm{~mm}$. b) $r=106,3 \mathrm{~mm}$. c) $r=99,6 \mathrm{~mm}$.

[Finite-length effects : eddy currents with $f_{\mathrm{r}}=1 \mathrm{~Hz}$. (- - $J_{r} ;(---) J_{\theta} ;(-) J_{z}$. a) $r=116.8 \mathrm{~mm}$. b) $r=106.3 \mathrm{~mm}$. c) $r=99.6 \mathrm{~mm}$.] 


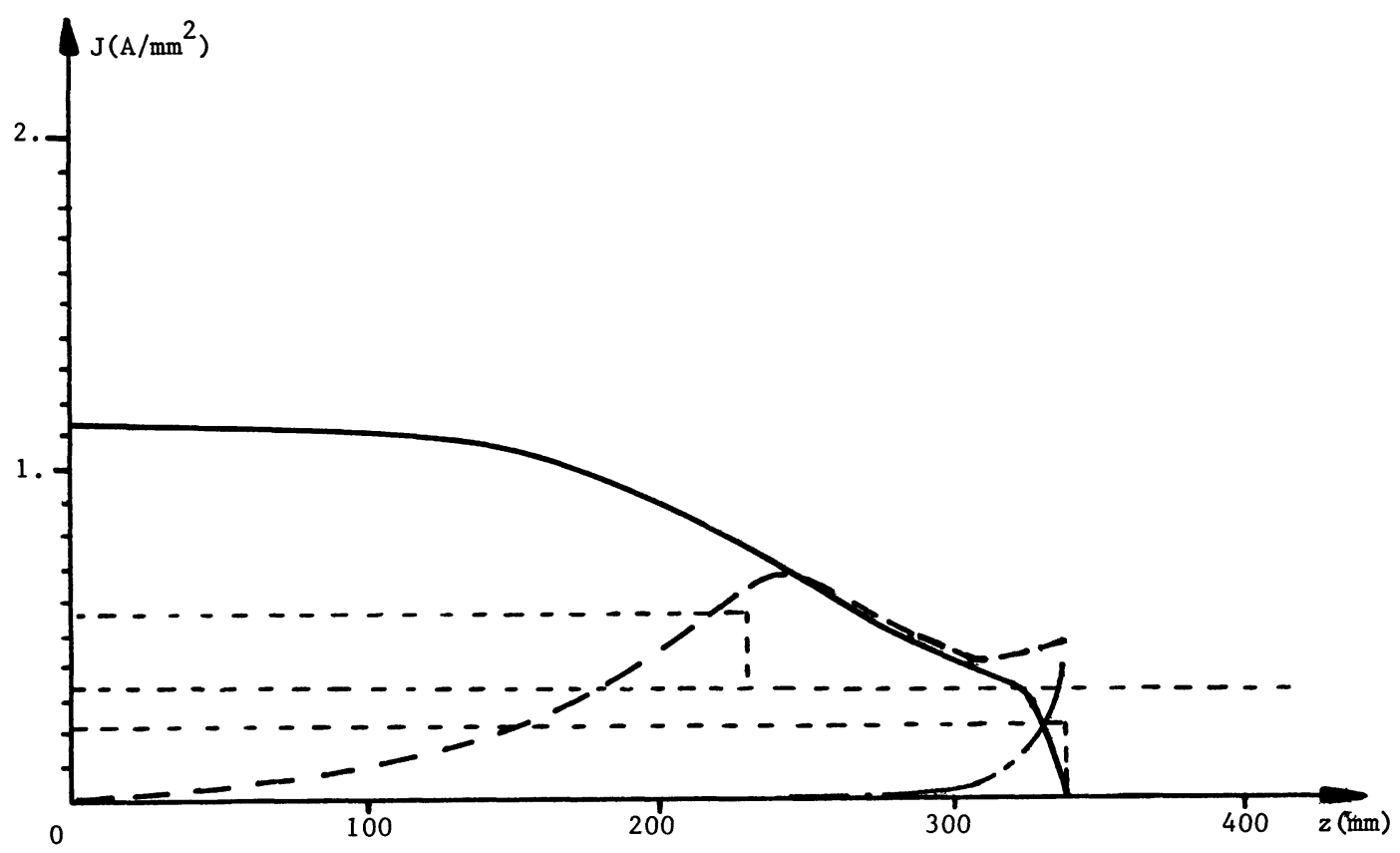

Fig. 6. (b)

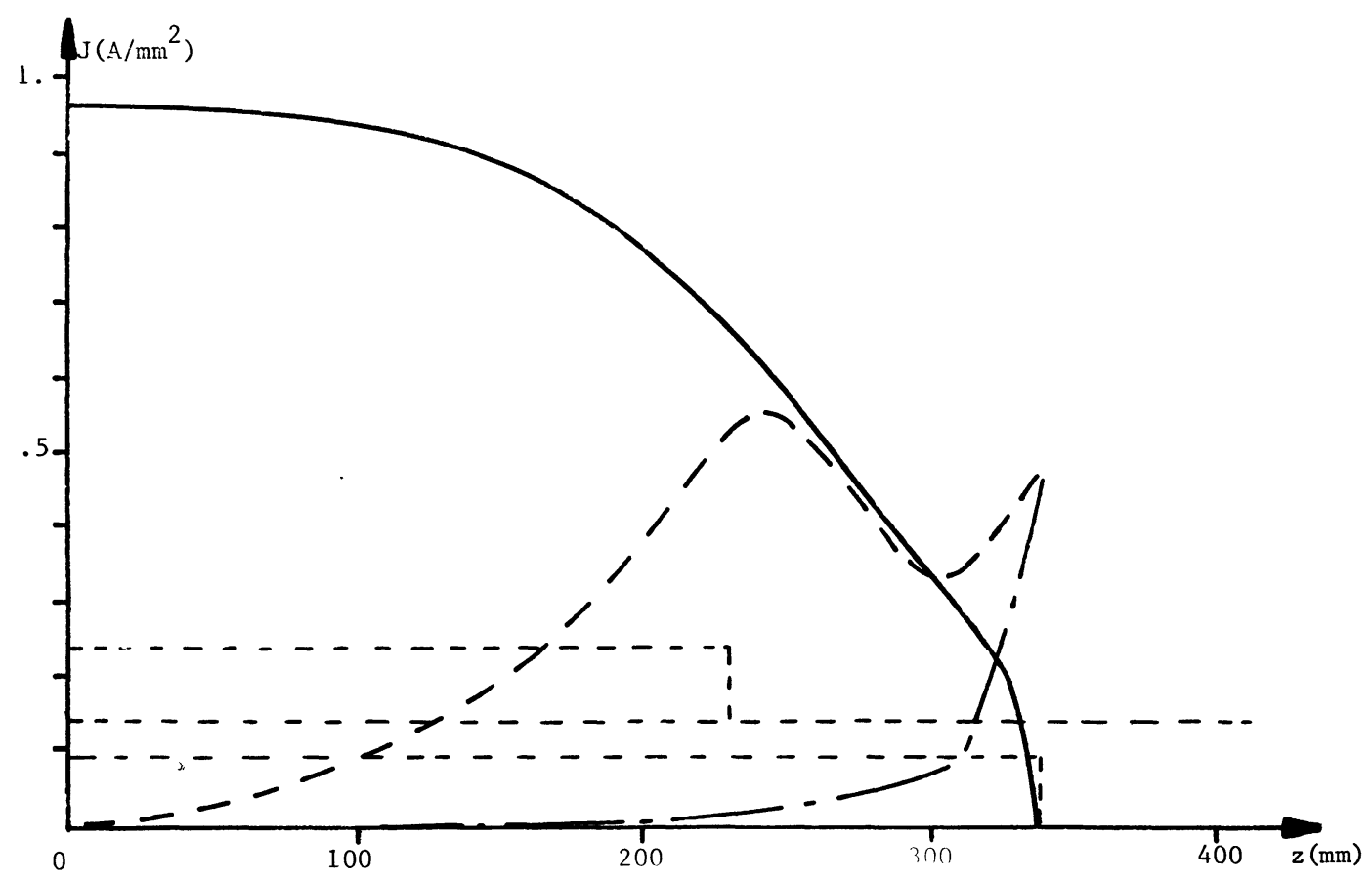

Fig. 6. (c)

raction des têtes de bobines et du rotor favorise la fermeture des courants induits à l'extérieur de la zone du stator, augmentant par là même leur composante utile $J_{z}$. C'est ce que confirme le tracé de la répartition des courants induits sur les figures 11 et 12. Ceci est bien sûr à pondérer dans la mesure où les têtes de bobines ont été modélisées concentriques au rotor pour simplifier l'acquisition des données, ce qui majore leur couplage magnétique avec les courants induits rotoriques.

\section{Conclusion.}

Nous avons présenté un modèle numérique pseudo3D bien adapté à la résolution de l'équation de diffusion non linéaire en régime harmonique. Ce modèle constitue une approche complémentaire des modèles 2D, car il permet de mieux cerner les phénomènes liés aux anisotropies axiales de structure. L'étude des effets d'extrémité dans un rotor massif en est un exemple significatif. Mais on aurait 

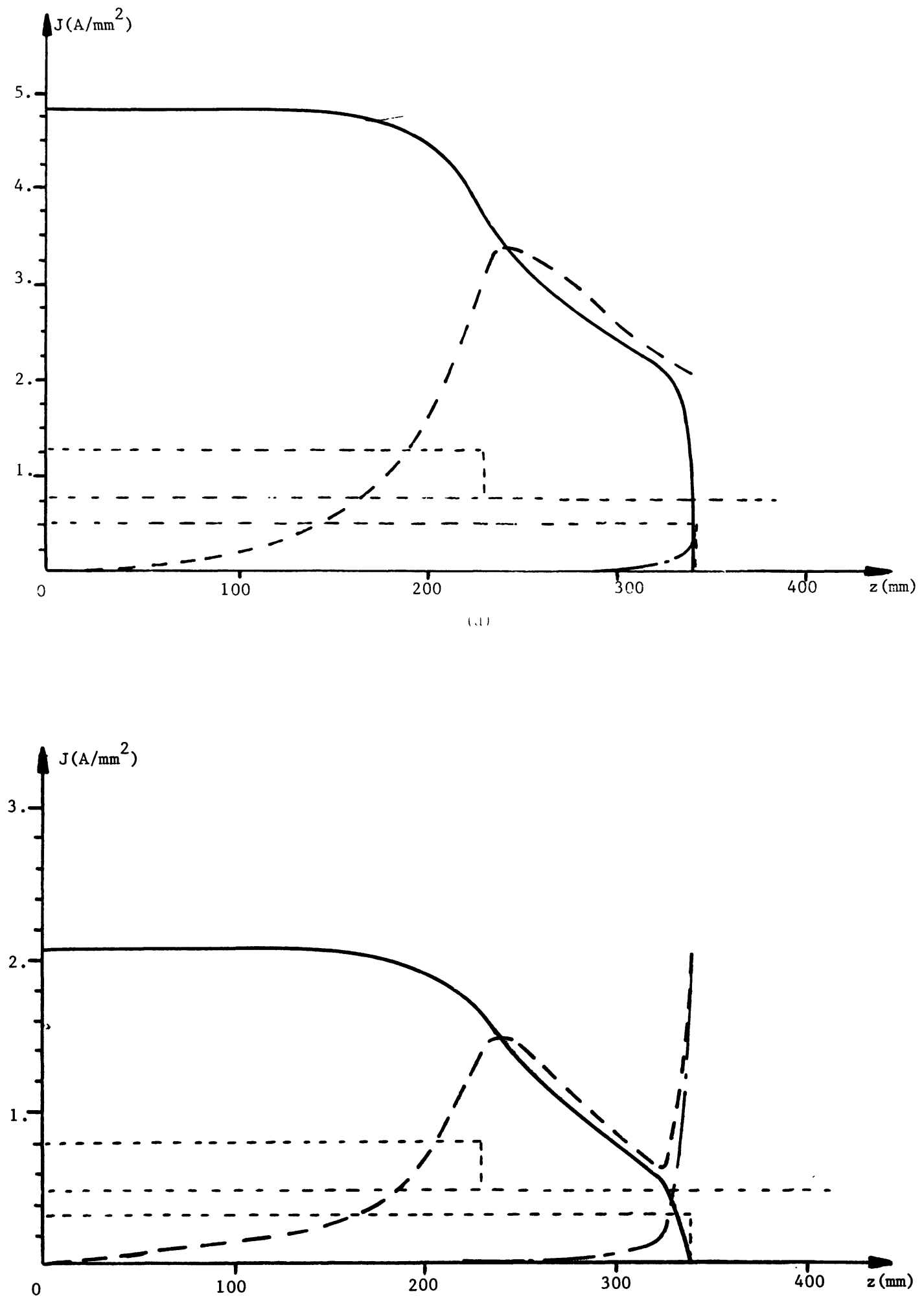

Fig. 7. - Effets de longueur finie : courants induits pour $f_{\mathrm{r}}=10 \mathrm{~Hz}$. (- - - $J_{r} ;(-\cdots) J_{\theta} ;\left(-(-) J_{z}\right.$ a) $r=116,8 \mathrm{~mm}$. b) $r=106,3 \mathrm{~mm}$. c) $r=99,6 \mathrm{~mm}$.

[Finite-length effects : eddy currents with $f_{\mathrm{r}}=10 \mathrm{~Hz}$. (-- -) $J_{r} ;(---) J_{\theta}$; (-) $J_{z}$, a) $r=116.8 \mathrm{~mm}$. b) $r=106.3 \mathrm{~mm}$. c) $r=99.6 \mathrm{~mm}$.] 


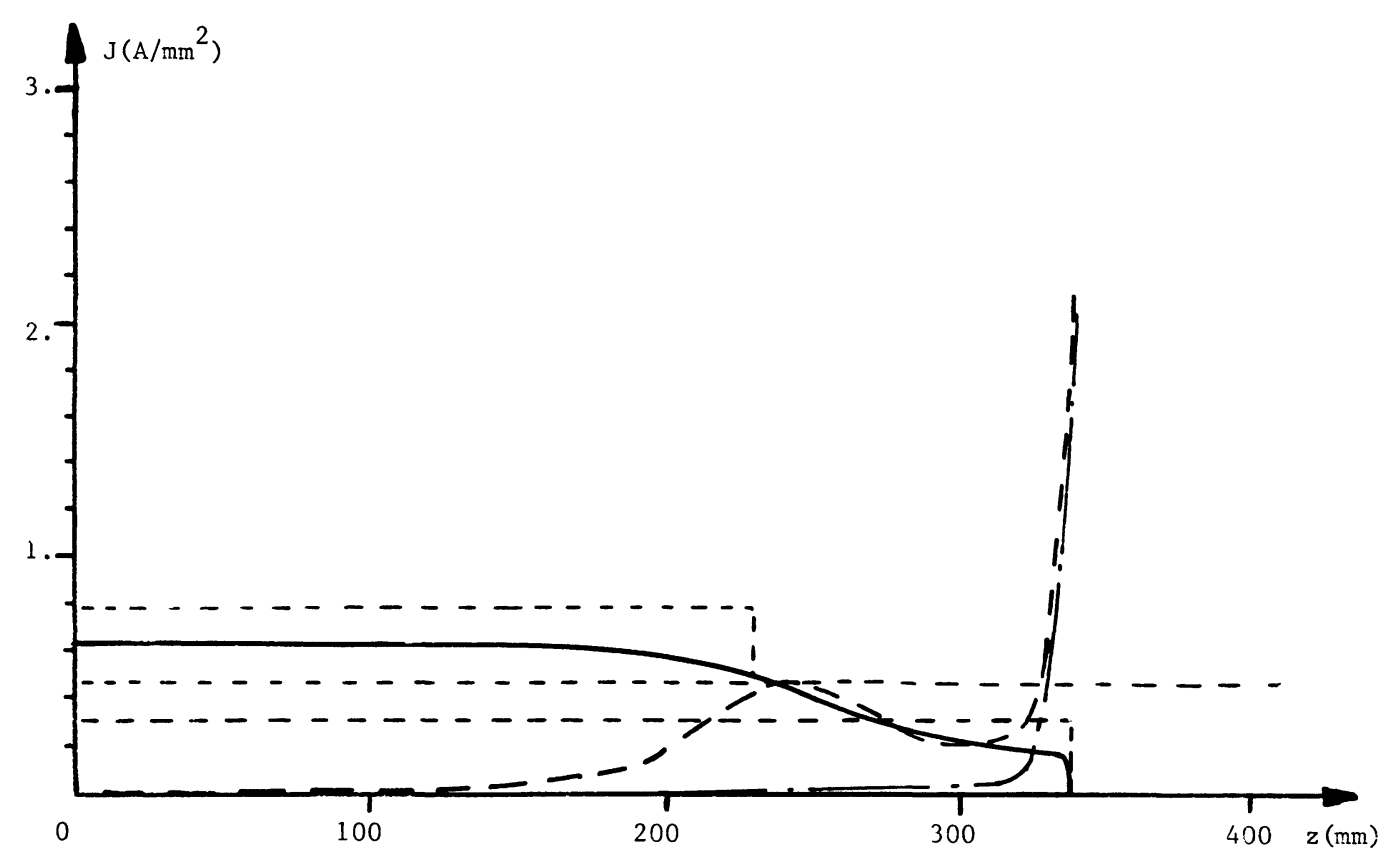

Fig. 7. (c)

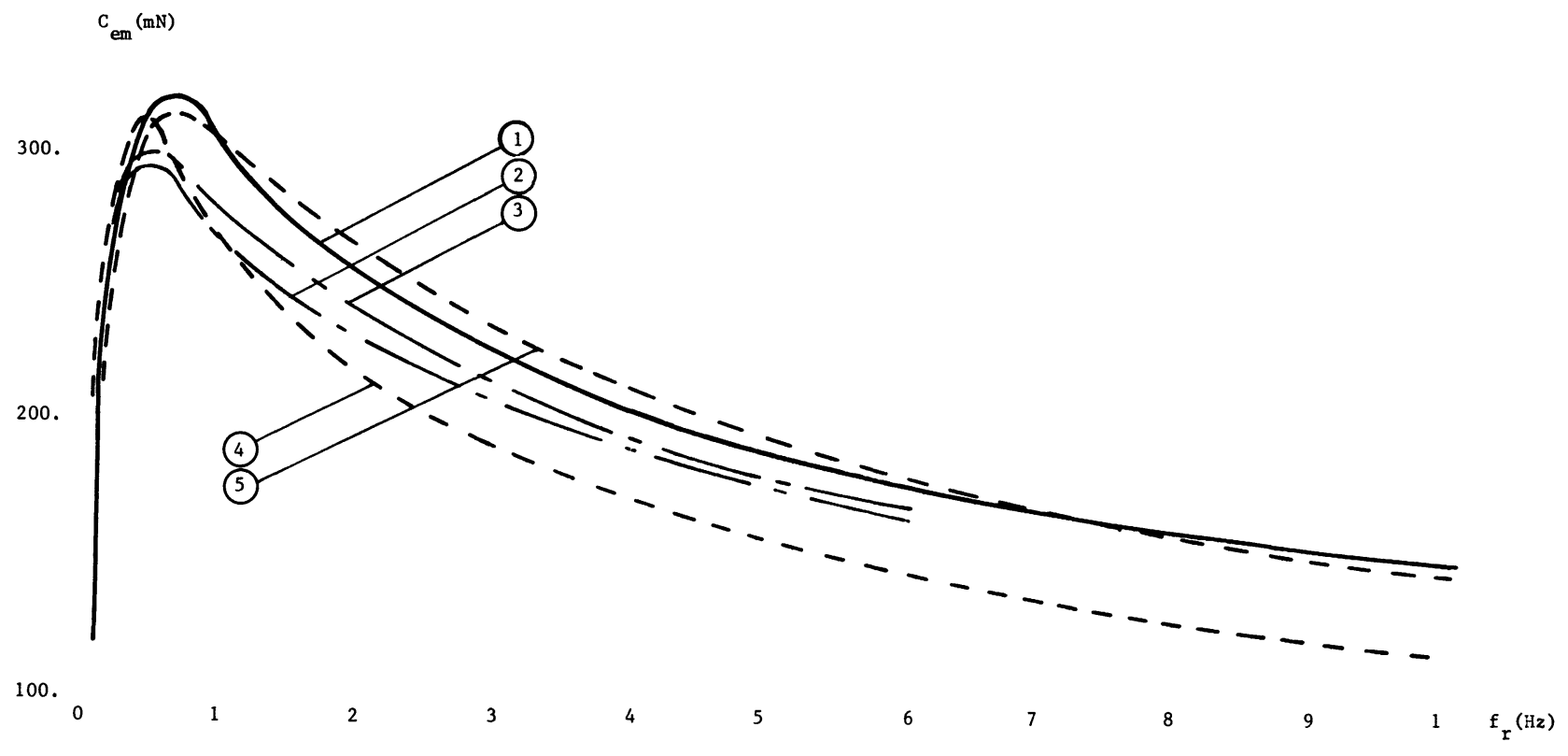

Fig. 8. - Effets de longueur finie : caractéristique couple/fréquence $f_{\mathrm{r}}$. Comparaison des modèles 2D et Pseudo-3D. 1) Modèle pseudo-3D. 2) Modèle pseudo-3D avec rotor raccourci. 3) Modèle pseudo-3D avec têtes de bobine raccourcies. 4) Modèle 2D - Conductivité du rotor non corrigée. 5) Modèle 2D - Conductivité du rotor corrigée.

[Finite-length effects : torque $/ f_{\mathrm{r}}$ frequency characteristics. Comparison of 2D and quasi-3D models. 1) Quasi-3D model. 2) Quasi-3D model with short rotor. 3) Quasi-3D model with short stator end windings. 4) $2 \mathrm{D}$ model - rotor conductivity no corrected. 5) 2D model — rotor conductivity corrected.] 


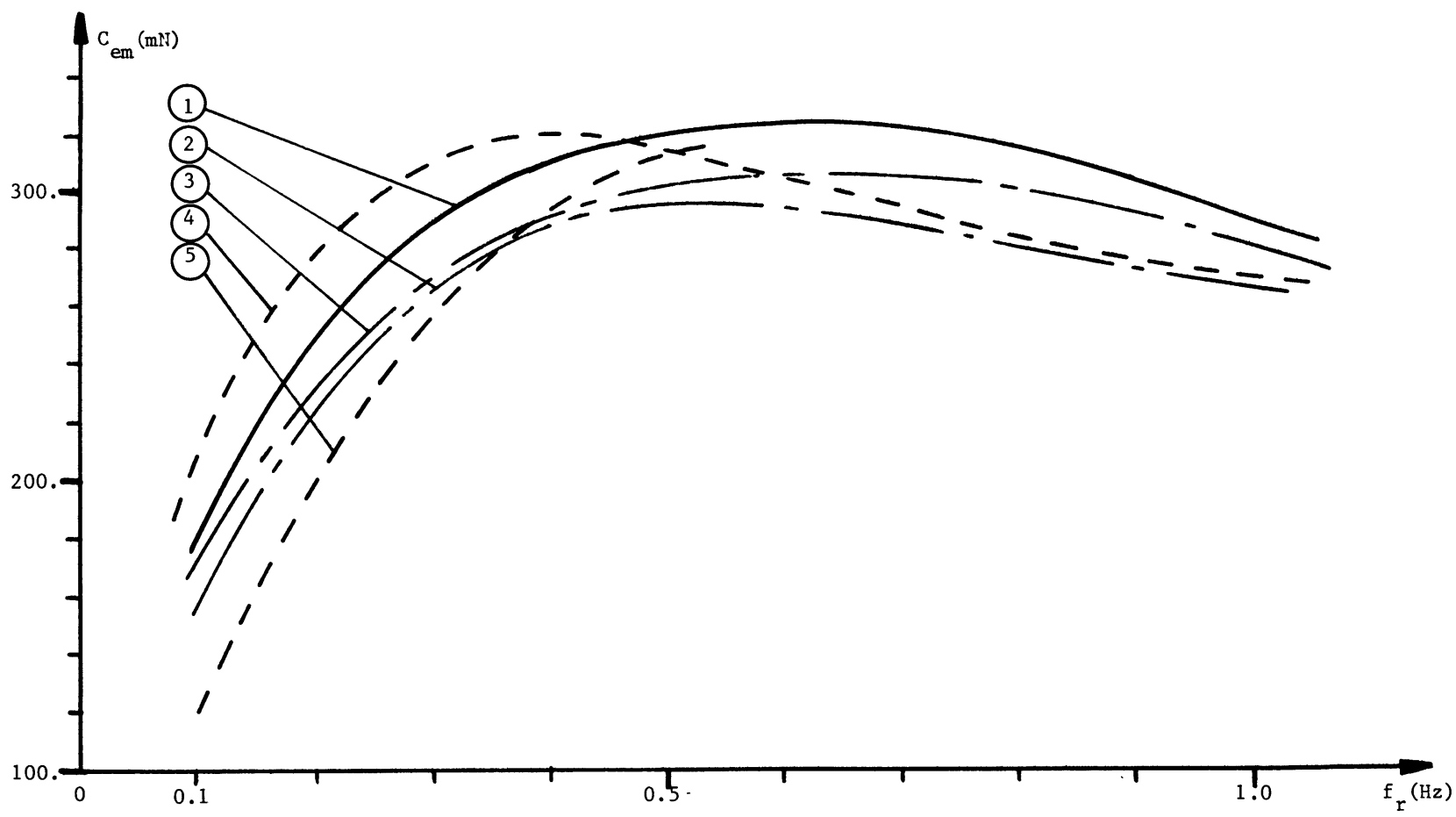

Fig. 9. - Effets de longueur finie : caractéristique couple/fréquence $f_{\mathrm{r}}$. Comparaison des modèles 2D et Pseudo-3D. 1) Modèle pseudo-3D. 2) Modèle pseudo-3D avec rotor raccourci. 3) Modèle pseudo-3D avec têtes de bobine raccourcies. 4) Modèle 2D - Conductivité du rotor non corrigée. 5) Modèle 2D - Conductivité du rotor corrigée.

[Finite-length effects : torque $/ f_{\mathrm{r}}$ frequency characteristics. Comparison of $2 \mathrm{D}$ and quasi-3D models. 1) Quasi-3D model. 2) Quasi-3D model with short rotor. 3) Quasi-3D model with short stator end windings. 4) $2 \mathrm{D}$ model - rotor conductivity no corrected. 5) 2D model - rotor conductivity corrected.]

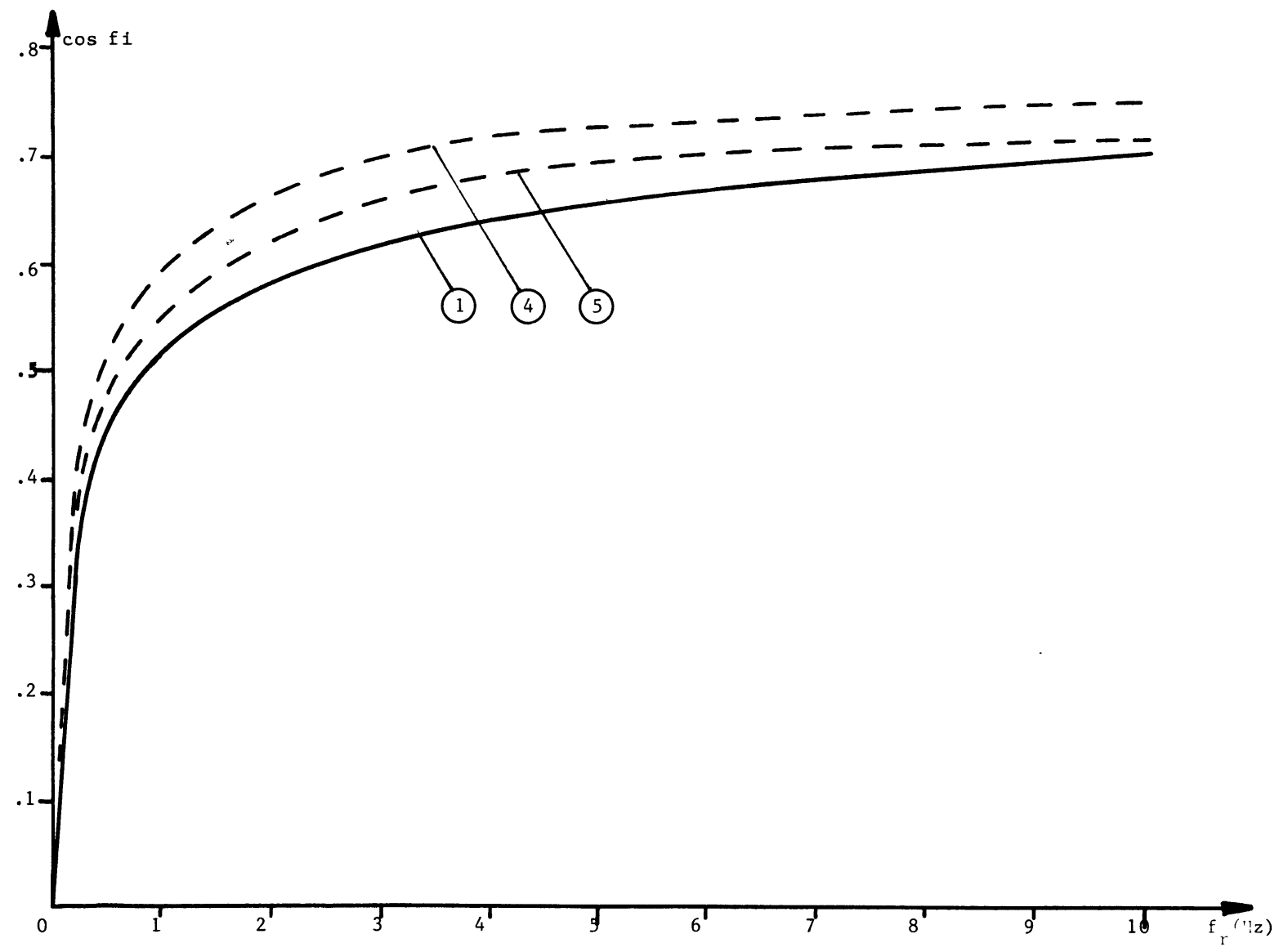




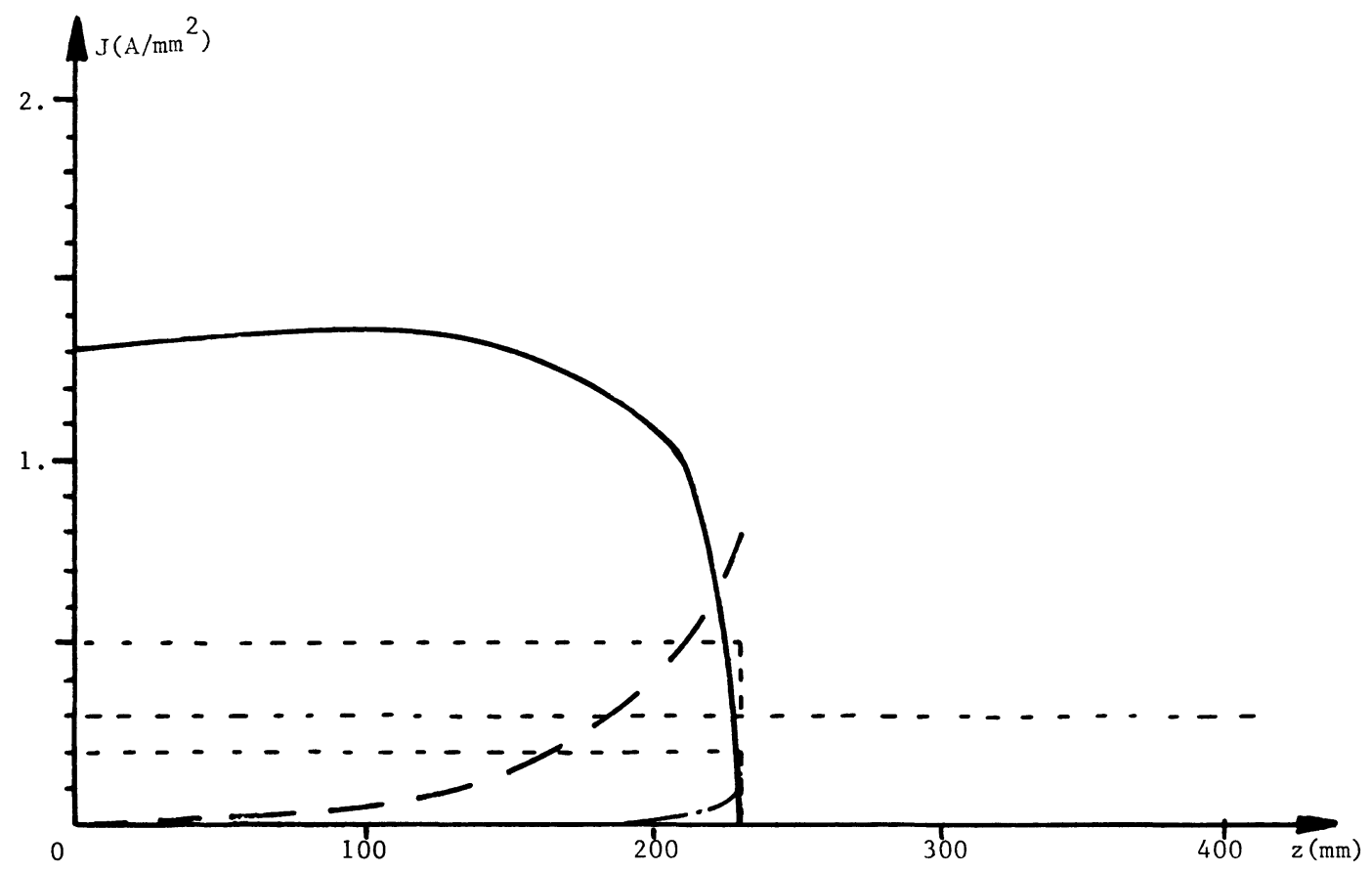

Fig. 11. - Effets de longueur finie : influence du raccourcissement du rotor sur les courants induits $(r=116,8 \mathrm{~mm})$. $(--$ - $) J_{r} ;(---) J_{\theta} ;(\longleftarrow) J_{z}$.

[Finite-length effects : eddy-current distribution with short rotor $(r=116.8 \mathrm{~mm}) .\left(--{ }^{\prime}\right) J_{r} ;(---) J_{\theta} ;(-\longrightarrow)$ $J_{z}$.]

$$
\mathrm{J}\left(\mathrm{A} / \mathrm{mm}^{2}\right)
$$

2.

1.

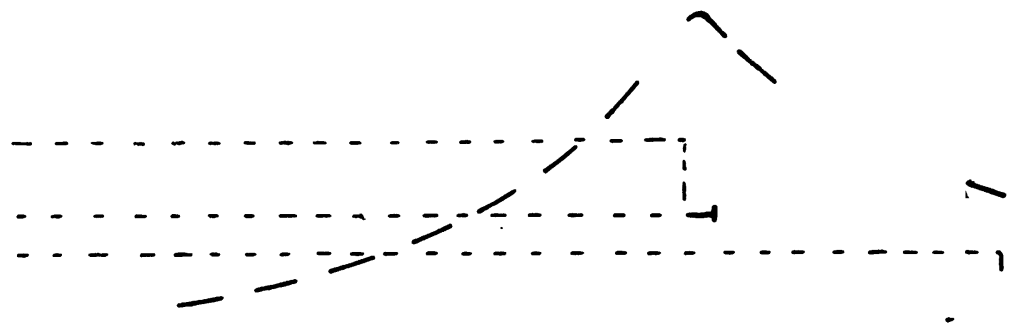

$\begin{array}{lllll}0 & 100 & 200 & 300 & \mathrm{z}(\mathrm{mm})\end{array}$

Fig. 12. - Effets de longueur finie: influence du raccourcissement des têtes de bobine sur les courants induits $(r=116,8 \mathrm{~mm})$. ( - - $J_{r} ;(---) J_{\theta} ;(\longrightarrow) J_{z}$.

[Finite-length effects : eddy-current distribution with short stator end windings $(r=116.8 \mathrm{~mm})$. (-- -) $J_{r}$; (---) $\left.J_{\theta} ;(\longrightarrow) J_{z} \cdot\right]$

Fig. 10. - Effets de longueur finie : caractéristique $\cos \varphi / f$. (Comparaison des modèles 2D et Pseudo-3D. 1) Modele pseudo-3D. 4) Modèle 2D - Conductivité du rotor non corrigée. 5) Modèle 2D - Conductivité du rotor corrigée.

[Finite-length effects : $\cos \varphi / f_{\mathrm{r}}$ frequency characteristics. Comparison of 2D and quasi-3D models. 1) Quasi-3D model. 4) $2 \mathrm{D}$ model - rotor conductivity no corrected. 5) $2 \mathrm{D}$ model — rotor conductivity corrected.] 
tout aussi bien pu l'utiliser pour traiter d'autres problèmes spécifiques à ce type de structure, comme le dimensionnement du rainurage circonférentiel pour réduire les pertes de surface liées à l'encochage axial du stator [7], ou encore le couplage entre les champs glissant et tournant dans un actionneur à deux degrés de liberté, au prix d'une légère modification de la fonction descriptive (37) des sources.

De plus son implémentation numérique est relativement aisée en comparaison des modèles 3D.

Enfin, grâce à la partition du domaine d'étude en zones à potentiels spécifíques, ce modèle peut être complété pour tenir compte de la nature laminaire des courants induits dans les tôles frontales de stator.

Toutes ces raisons donnent à penser que l'utilisation combinée des modèles $2 \mathrm{D}$ et pseudo-3D devrait permettre de réserver l'usage des modèles $3 \mathrm{D}$ à l'étude de phénomènes très spécifiques, fortement liés au couplage des anisotropies axiales et circonférentielles.

\section{Bibliographie}

[1] Wiart A., Gaussin G., Marchenoir A., Machine homopolaire alimentée par onduleur de courant pour entraînement de grande puissance, R.G.E. 10 (1984) 666-672.

[2] BRATOlJiC T., Influence of grooves on the eddycurrent loss in solid rotors, Int. Conf. On Elec. Mach., GP 1/9, Athènes (1980) pp. 1560-1572.

[3] Dorairaj K. R., Krishnamurthy M. R., Polyphase induction machine with a slitted ferromagnetic rotor - II - Analysis, IEEE PAS-86 (1967) 844-855.

[4] Woolley I., Chalmers B. J., Internal design of unlaminated-rotor induction machines, Proc. IEE 121 (1974) 197-202.

[5] KANT M., Machines à deux degrés de liberté, R.G.E. 10 (1984) 642-647.

[6] NAKATA T., Comparison of various methods for 3D eddy current analysis, IEEE MAG-24 (1988) 3159-3161.

[7] Biedinger J. M., Contribution à l'étude de la diffusion du champ électromagnétique dans le fer massif. Application à l'analyse d'un moteur asynchrone à rotor massif, Thèse de Doctorat d'Etat, Université de Compiègne (1986).

[8] JACK A. G., Mecrow B. C., Calculation of threedimensional electromagnetic fields involving laminar eddy currents, Proc. IEE 134 (1987) 663-671.

[9] Morse P. M., FeshbaCh H., Methods of theoretical physics (McGraw-Hill) 1955.

[10] Driga M. D., Magnetic field diffusion in fast discharging homopolar machines, Elec. Mach. Electrom. 2 (1977) 49-60.

[11] Renhart W., Stogner H., Preis K., Calculation of 3D eddy current problems by finite element method using either an electric or a magnetic vector potential, IEEE MAG-24 (1988) 122-125.
[12] Leonard P. J., Rodger D., Finite element scheme for transient 3D eddy currents, IEEE MAG-24 (1988) 90-93.

[13] Emson C.R.I., Simkin J., An optimal method for 3D eddy currents, IEEE MAG-19 (1983) 24502452.

[14] Luomi J., NiemenmaA A., Arkkio A., On the use of effective reluctivities in magnetic field analysis of induction motors fed from a sinusoïdal voltage source, ICEM, Münich, Pt 2 (1986) pp. 706-711.

[15] Coulomb J. L., Analyse tridimensionnelle des champs électriques et magnétiques par la méthode des éléments finis. Thèse de Doctorat d'Etat, Grenoble (1981).

[16] Carpenter C. J., Locke D. H., Numerical models of three-dimensional end winding arrays. Compumag (1976) pp. 47-55.

[17] Dhatt G., Touzot G., Une présentation de la méthode des éléments finis. Collection Université de Compiègne, Maloine SA éditeur (1981).

[18] Yee H., Wilson T., Saturation and finite-length effects in solid-rotor induction machine, Proc. IEE 119 (1972) 877-882.

[19] Woolley I., Chalmers B. J., End effects in unlaminated-rotor induction machines, Proc. IEE 120 (1973) 641-646.

[20] Sikorba R. et al., Analysis of the magnetic field in the end region of induction motor, IEEE MAG18 (1982) 674-678.

[21] Demerdash N. A., Nehl T. W., Use of numerical analysis of nonlinear eddy current problems by finite elements in the determination of parameters of electrical machines with solid iron rotors, IEEE MAG-15 (1979) 1482-1484.

[22] DU Terrail Y. et al., Nonlinear complex finite elements analysis of electromagnetic field in steady-state AC devices, IEEE MAG-20 (1984) 549-552. 